\title{
Voltage-Gated Potassium Channels Ensure Action Potential Shape Fidelity in Distal Axons
}

\author{
Victoria Gonzalez Sabater, ${ }^{1,2 *}$ Mark Rigby, ${ }^{1,2 *}$ and ${ }^{-J u a n}$ Burrone $^{1,2}$ \\ ${ }^{1}$ MRC Centre for Neurodevelopmental Disorders, Institute of Psychiatry, Psychology and Neuroscience, King's College London, London SE1 1UL, \\ United Kingdom, and ${ }^{2}$ Centre for Developmental Neurobiology, Institute of Psychiatry, Psychology and Neuroscience, King's College London, \\ London SE1 1UL, United Kingdom
}

The initiation and propagation of the action potential (AP) along an axon allows neurons to convey information rapidly and across distant sites. Although AP properties have typically been characterized at the soma and proximal axon, knowledge of the propagation of APs toward distal axonal domains of mammalian CNS neurons remains limited. We used genetically encoded voltage indicators (GEVIs) to image APs with submillisecond temporal resolution simultaneously at different locations along the long axons of dissociated hippocampal neurons from rat embryos of either sex. We found that APs became sharper and showed remarkable fidelity as they traveled toward distal axons, even during a high-frequency train. Blocking voltage-gated potassium channels $\left(\mathrm{K}_{\mathrm{v}}\right)$ with 4-AP resulted in an increase in AP width in all compartments, which was stronger at distal locations and exacerbated during AP trains. We conclude that the higher levels of Kv channel activity in distal axons serve to sustain AP fidelity, conveying a reliable digital signal to presynaptic boutons.

Key words: action potential; axon; potassium channels; voltage imaging

\section{Significance Statement}

The AP represents the electrical signal carried along axons toward distant presynaptic boutons where it culminates in the release of neurotransmitters. The nonlinearities involved in this process are such that small changes in AP shape can result in large changes in neurotransmitter release. Since axons are remarkably long structures, any distortions that APs suffer along the way have the potential to translate into a significant modulation of synaptic transmission, particularly in distal domains. To avoid these issues, distal axons have ensured that signals are kept remarkably constant and insensitive to modulation during a train, despite the long distances traveled. Here, we uncover the mechanisms that allow distal axonal domains to provide a reliable and faithful digital signal to presynaptic terminals.

\section{Introduction}

Projection neurons in the CNS extend long axons often forming thousands of small en passant synaptic boutons, hundreds of microns from their cell soma (Debanne et al., 2011). The

\footnotetext{
Received Oct. 30, 2020; revised Mar. 29, 2021; accepted Apr. 1, 2021.

Author contributions: V.G.S., M.R., and J.B. designed research; V.G.S. and M.R. performed research; V.G.S analyzed data; V.G.S., M.R., and J.B. wrote the paper.

This work was supported by a Wellcome Trust Investigator Award (095589/Z/11/Z), European Research Council Starting Grant 282047 to J.B., a Medical Research Council studentship to V.G.S., and a National Centre for the Replacement, Refinement, \& Reduction of Animals in Research Training Fellowship to M.R. We thank Thomas Knöpfel, Martin Meyer, and Matthew Grubb for discussions and suggestions; all the members of the Juan Burrone Lab for feedback and comments; and Kiryl Piatkevich for the expression plasmids for both Archon 1 and 2.

*V.G.S. and M.R. contributed equally to this work.

The authors declare no competing interests.

Correspondence should be addressed to Juan Burrone at juan.burrone@kcl.ac.uk.

https://doi.org/10.1523/JNEUROSCI.2765-20.2021

Copyright $\odot 2021$ Gonzalez Sabater, Rigby et al.

This is an open-access article distributed under the terms of the Creative Commons Attribution 4.0 International license, which permits unrestricted use, distribution and reproduction in any medium provided that the original work is properly attributed.
}

probability that neurotransmitter is released from such boutons depends on a number of factors set in motion by an action potential (Branco and Staras, 2009; Dittman and Ryan, 2019; Kawaguchi, 2019). Modest changes in AP waveform can strongly influence voltage-gated $\mathrm{Ca}^{2+}$ channel open probability (Sabatini and Regehr, 1997; Scarnati et al., 2020), the subsequent driving force for $\mathrm{Ca}^{2+}$ entry (Scarnati et al., 2020), and given its highly nonlinear calcium dependence (Schneggenburger and Neher, 2000; Neher and Sakaba, 2008), release probability $\left(P_{r}\right)$ (Rama et al., 2015). A model of synaptic transmission from distally located projection neuron release sites therefore requires knowledge of AP propagation and waveform.

The AP is not an immutable waveform from initiation to termination but varies as it propagates along the axon (Branco and Staras, 2009; Debanne et al., 2011; Scarnati et al., 2020). Localized changes in axon morphology, as well as Nav and $\mathrm{Kv}$ channel expression and modulation, alter the AP waveform as it propagates into the soma and distally through the axonal tree (Goldstein and Rall, 1974; Geiger and Jonas, 2000; Kole et al., 
2007; Hoppa et al., 2014; Cho et al., 2017). Such differences locally affect axonal AP propagation fidelity (Khaliq and Raman, 2005; Monsivais et al., 2005; Sasaki et al., 2012; Kawaguchi and Sakaba, 2015; Cho et al., 2017), speed (Chéreau et al., 2017), and shape (Kole et al., 2007; Alle et al., 2011; Hoppa et al., 2014). It is therefore important to understand both the spatial and temporal modulation of the axonal APs during physiological firing regimes.

The localized heterogeneity in axonal AP properties ideally requires the capacity to record the $\mathrm{AP}$ at multiple locations. Pipette-based recordings offer unparalleled temporal and voltage sensitivity, but the small size of the axon generally stipulates technically challenging, single-site measurements (Novak et al., 2013; Kawaguchi and Sakaba, 2017; Vivekananda et al., 2017; Ritzau-Jost et al., 2021). Given that whole-cell patch-clamp techniques both dialyze the internal cytosol and rupture large parts of the membrane under study, alternative higher throughput approaches are worth consideration.

Genetically encoded voltage indicators (GEVIs) potentially provide a means to circumvent some of the pitfalls of patchclamp recordings (Knöpfel and Song, 2019; Panzera and Hoppa, 2019). Of their growing number, the opsin-based family is among the most suitable to record the axonal AP. The relatively fast on/off kinetics and high voltage sensitivity have been used to detect differences in axonal AP waveform (Kralj et al., 2011; Hochbaum et al., 2014; Hoppa et al., 2014; Cho et al., 2020). However, low quantum efficiency and brightness stipulate very high illumination intensities, especially in the axon where small volumes of plasma membrane limit the capacity to hold expressed proteins. More recently developed opsin-based GEVIs demonstrate improved brightness but have not been validated beyond their original reports. Of note, Archon2 exhibited comparable response kinetics but was severalfold brighter and responsive to voltage changes (Piatkevich et al., 2018). In addition, the FRET-opsin-based sensor Ace $2 \mathrm{~N}-4 \mathrm{AA}-\mathrm{mNe}$ n took advantage of the large quantum efficiency and photostability of $\mathrm{mNeonGreen}$ to improve on previous issues of probe brightness (Gong et al., 2015).

Based on their reported brightness and kinetic properties, Ace 2N-4AA-mNeon and Archon2 were compared for their ability to report the AP waveform and propagation along an axon. By examining local properties of the axonal, we show that AP width sharpens toward distal axonal domains and that the AP waveform remained largely unaltered during a $20 \mathrm{~Hz}$ AP train in distal but not in proximal axons or in the soma. Furthermore, we show that Kv channels in distal axons limit the width and amplitude of an AP, thereby improving AP reliability during high-frequency trains.

\section{Materials and Methods}

Hippocampal neuronal cultures and transfection. Hippocampi were dissected from embryonic day 17.5 Wistar rat pups of either sex, treated with trypsin (Worthington) at $0.5 \mathrm{mg} / \mathrm{ml}$, and mechanically dissociated using fire polished Pasteur pipettes. Neurons were plated on $18 \mathrm{~mm}$ glass coverslips (Thermo Fisher Scientific) pretreated with $100 \mu \mathrm{g} / \mathrm{ml}$ poly-Llysine (Sigma) and coated with $10 \mu \mathrm{g} / \mathrm{ml}$ laminin (Life Technologies). Cultures were maintained in Neurobasal medium (Life Technologies) with B27 (1×; Invitrogen) and GlutaMAX (1×; Life Technologies), supplemented with fetal bovine serum (FBS; $2 \%$; Biosera) and penicillin/ streptomycin (1\%; Sigma), at $37^{\circ} \mathrm{C}$ in a humidified incubator with $5 \%$ $\mathrm{CO}_{2}$.

For transfections with Ace2N-mNeon-4AA (Ace-mNeon; Biolife; Gong et al., 2015), the Effectene transfection reagent (Qiagen) was used.
The medium was changed at $3 \mathrm{~d}$ in vitro (DIV) to culture medium without antibiotic or FBS, and transfections with Effectene were performed at DIV 7 following the manufacturer's protocol. After transfection, neurons were maintained in serum-free media without antibiotics. For transfections with Archon2 (Piatkevich et al., 2018; gift from E. Boyden laboratory), the calcium-phosphate method (Ca-Phos) was used. DIV 35 neurons were transfected following an adapted version of a low-toxicity protocol for low-density cultures (Jiang and Chen, 2006). The coverslips were returned to their original culture medium with FBS for two more days before changing to serum-free media without antibiotics.

For all experiments, $30 \%$ of the medium was changed weekly, and neurons were imaged 7-14 d after transfection (14-21 DIV).

Live-cell recording and imaging conditions. Neurons were imaged using an inverted Olympus IX71 epifluorescence microscope with a $60 \times$ 1.42 NA oil-immersion objective. Coverslips were mounted in a heated chamber (total volume $\sim 500 \mu \mathrm{L}$; Warner Instruments) and placed on an inverted movable top plate microscope stage (Scientifica). Cells were maintained in external HEPES-buffered saline solution [HBS; containing the following (in mM): $2 \mathrm{CaCl}_{2}, 1.6 \mathrm{MgCl}_{2}, 1.45 \mathrm{NaCl}, 2.5 \mathrm{KCl}, 10$ glucose, 10 HEPES, $\mathrm{pH}=7.4$, osmolarity $=290 \mathrm{mOsm}]$. For experiments at physiological temperature, the chamber was heated to $32-35^{\circ} \mathrm{C}$, and the $\mathrm{pH}$ of the HBS solution was adjusted for this temperature.

Current-clamp recordings were made in the whole-cell configuration from the soma of visually identified transfected neurons at room temperature. Recordings were performed with borosilicate glass pipettes pulled to a resistance of 4-6 M $\Omega$, fire polished, and filled with internal solution containing the following (in mM): $125 \mathrm{KMeSO}_{4}, 5 \mathrm{MgCl}_{2}, 10$ EGTA, 10 HEPES, $0.5 \mathrm{NaGTP} 5 \mathrm{Na}_{2} \mathrm{ATP}, \mathrm{pH}=7.4$. Data were acquired with a MultiClamp 700B amplifier (Molecular Devices), and digitized with a Digidata 1440A digitizer (Molecular Devices) at a sampling rate of $20 \mathrm{kHz}$. Recordings were acquired using Clampex version 10.3 (Molecular Devices) with a gain value of 5 and a Bessel filter set to $10 \mathrm{kHz}$. Pipette capacitance neutralization and bridge balance were applied.

For Ace-mNeon imaging experiments, excitation illumination was provided using a $525 \mathrm{~nm}$ LED (Solis), a 500/20 nm excitation filter, and a $510 \mathrm{~nm}$ long-pass dichroic mirror. Ace-mNeon emission transmitted through the dichroic was filtered using a $520 \mathrm{~nm}$ long-pass filters (Chroma Technology). A power density of $10 \mathrm{~mW} / \mathrm{mm}^{2}$ was obtained at the specimen plane. Excitation of Archon2 was achieved using a $635 \mathrm{~nm}$ diode laser (MRL-III-635L >200 mW <1\% RMS; ReadyLasers), expanded threefold using a custom Galilean beam expander and focused onto the back aperture of the objective. Excitation illumination passed through a $640 / 30 \mathrm{~nm}$ excitation filter and a $660 \mathrm{~nm}$ long-pass dichroic; emitted fluorescence was filtered using a $690 / 50 \mathrm{~nm}$ filter (Chroma Technology). The power density achieved for Archon2 imaging was 2.6 $\mathrm{W} / \mathrm{mm}^{2}$ at the specimen plane.

Images were acquired at $3.2 \mathrm{kHz}$ with an ORCA-Flash4.0 V2 C1144022CU scientific CMOS camera (Hamamatsu Photonics) cooled to approximately $-20^{\circ} \mathrm{C}$ with the Exos2 water cooling system (Koolance). Images were acquired with HCImage software (Hamamatsu Photonics), binned to $4 \times 4$ and cropped to a $16 \times 512$ pixel region of interest, necessary to achieve the high image acquisition rates. Images were saved in CXD format. For the reconstruction of the axonal arbor after the experiment, high-resolution $2048 \times 2048$ images were acquired with an exposure of $100 \mathrm{~ms}$.

Unless paired with whole-cell patch-clamp recordings, where AP stimulation was achieved through the patch pipette, stimulation within imaging experiments was achieved with an extracellular tungsten parallel bipolar electrode (FHC) mounted on a PatchStar Motorized Micromanipulator (Scientifica). For experiments with axonal imaging only, $1 \mathrm{~ms}$ pulses of $10 \mathrm{mV}$ were delivered, and $50 \mu \mathrm{s}$ pulses of $30 \mathrm{mV}$ were applied for experiments involving both somatic and axonal imaging. Shorter pulses were required when imaging the somatic AP to keep the stimulation pulse artefact from contaminating the AP signal.

The timing of the LEDs and laser, acquisition, and stimulation were triggered externally through Clampex software (pClamp 10, Molecular Devices). 
Unless otherwise stated, all recordings were performed in the presence of NBQX (10 $\mu \mathrm{m}$; Tocris Bioscience), Gabazine (10 $\mu \mathrm{m}$; Tocris Bioscience) and D-2-amino-5-phosphonovalerate (25 $\mu \mathrm{M}$; Tocris Bioscience) to block synaptic transmission and ensure that the observed events were because of the stimulation only. When necessary, 4-aminopyrimidine (4-ap; $30 \mu \mathrm{m}$; Tocris Bioscience) and/or Tetrodotoxin (TTX; $40 \mathrm{~nm}$; Tocris Bioscience) were supplied using a custom gravity-fed perfusion system in which bath volume was maintained by a peristaltic pump (120S, Watson-Marlow).

Electrophysiology and GEVI recording analysis. Analysis of patchclamp recordings was performed with custom software written in MATLAB (MathWorks). Values of cell capacitance, input resistance, and series resistance were estimated from membrane test recordings performed at regular intervals. All cells with a series resistance $>30 \mathrm{M} \Omega$ were discarded or ceased to be recorded from. Current-clamp recordings of APs in the soma were aligned to the AP peak, averaged, and the APs were analyzed to extract values for amplitude, onset, full-width at halfmaximum (width), $20-80 \%$ rise time, and $80-20 \%$ decay time, measured relative to baseline membrane potential.

Voltage imaging recordings were analyzed in ImageJ and custom scripts within MATLAB software as follows. Before analysis, if drift had occurred in the $x$ axis and $y$ axis during the recording, the images were aligned using the TurboReg ImageJ plugin (Thévenaz et al., 1998). Images were then imported into MATLAB, and fluorescence intensity profiles were obtained from regions of interest (ROIs) manually drawn around neuronal structures identified visually on a maximum projection image of the time series by averaging across the ROI pixels in each frame. The fluorescence profile was separated into single trials and corrected for background camera noise by subtracting the average intensity value of the time points where no illumination light was applied. Then, recordings were reconstructed at a sampling rate of $100 \mathrm{kHz}$ using cubic spline interpolation. Bleaching was estimated by fitting a single exponential function to each individual trace smoothed with an averaging filter with a window of three and interpolated. The resulting curve was used to correct an unfiltered interpolated version of the recording. An accurate representation of the AP waveform with an enhanced signal-tonoise ratio (SNR) was obtained by averaging over the repeats and extracting the AP parameters from the resulting trace. Nonaligned averages were used for analysis except for dual patch-clamp and voltage imaging experiments, where the peak of the electrophysiological recording could be used as reference for alignment. The parameters of the AP waveform measured were amplitude $(\Delta \mathrm{F} / \mathrm{F}), \mathrm{SNR}$, width, $20-80 \%$ rise time, $80-20 \%$ decay time, and decay time constant. The fluorescence profile during the $20 \mathrm{~ms}$ preceding a response was used as baseline for the calculations. The SNR was calculated as the ratio of the response amplitude to the SD of the baseline. For some experiments, the coefficient of variation was calculated as the ratio of the $\mathrm{SD}$ of the variable to its mean. The bleaching rate was estimated from the raw fluorescence time profile excluding the time points with no illumination.

The axonal arbor reconstruction was done in ImageJ. Overlapping images were stitched together using the MosaicJ plugin, and the axon was then traced with the NeuronJ plugin.

Experimental design and statistical analysis. Statistical analysis was performed using GraphPad Prism 8. All data are presented as the mean \pm SEM. Datasets were not assumed to be normally distributed unless the data passed a D'Agostino normality test. Results were considered significant at $p<0.05$. Please refer to figures for experimental design and statistical analysis details of each individual experiment.

\section{Results}

\section{Measuring the AP waveform with GEVIs}

To accurately perform GEVI-based measurements of the AP along the axons of dissociated hippocampal CA1 pyramidal neurons, we first assessed the ability of the selected GEVIs, Ace-2N4AA-mNeon and Archon2, to reliably report AP waveform at the soma. Somatic APs were evoked by current injection using a patch pipette, and the resulting APs were recorded in the whole- cell current-clamp configuration. At the same time, the fast changes in GEVI fluorescence expected from an AP signal were imaged at a high frame rate $(3.2 \mathrm{kHz})$ in a subsection of the somatic membrane (Fig. $1 A$ ). For both indicators, APs imaged at the soma could be reliably detected in single trials (Fig. 1B). When averaged across 20 trials, the imaged AP traces for both GEVIs closely resembled the electrophysiologically recorded AP waveform, even reporting small changes in voltage such as the slow subthreshold voltage rise preceding the AP (Fig. $1 B, B^{\prime}, C$, $\left.C^{\prime}\right)$. In general, we found that Archon2 displayed a larger change in fluorescence to single APs than Ace-mNeon $(\% \Delta \mathrm{F} / \mathrm{F}, 10 \%$ and $14 \%$ for Ace-mNeon and Archon2, respectively; $p<0.001 t$ test), whereas Ace-mNeon showed a higher SNR (88.9 \pm 16 and $43.8 \pm 3.7$ for Ace-mNeon and Archon2, respectively; $p<0.05$; Mann-Whitney test; Fig. 1J). Notably, Ace-mNeon displayed a small residual photocurrent in response to illumination, characterized by an initial depolarizing transient in membrane voltage $(\mathrm{Vm})$ that stabilized to a smaller depolarizing steady-state $\mathrm{Vm}$ offset within a few hundred milliseconds $[1.51 \mathrm{mV}$ over the resting membrane potential; confidence interval $(\mathrm{CI})=0.5,2.7 ; p<$ 0.001; one-sample Wilcoxon test; Fig. $1 K]$. To avoid the transient change in $\mathrm{Vm}$ from contaminating the optical AP signal we proceeded to elicit somatic APs with current injections delivered $500 \mathrm{~ms}$ after illumination onset when a steady-state depolarization had been reached. No changes in conductance were observed for Archon2-expressing neurons on illumination $\left(635 \mathrm{~nm}\right.$ laser at $2.6 \mathrm{~W} / \mathrm{mm}^{2}$; Fig. $1 K$; one-sample Wilcoxon tests).

We next examined how the main features of the AP waveform compared across different cells when measured with GEVIs and electrophysiology. For both Ace-mNeon and Archon2, we found that optically recorded measures of AP width and decay time were correlated with the current-clamp recorded AP, albeit with a small overestimation of the apparent AP kinetics (Fig. 1D,E,G,H). The overestimation was larger for Ace than for Archon2, most likely because of the faster reported kinetics of Archon2 ( Gong et al., 2015; Piatkevich et al., 2018; Fig. $1 L, M)$. The AP amplitude showed no correlation between GEVI and whole-cell current-clamp recordings, suggesting that optically acquired amplitude values cannot be compared across cells (Fig. 1F,I). A likely explanation for this result is the possible heterogeneity in baseline fluorescence levels resulting from GEVI molecules present within inner membranes (i.e., not properly targeted to the plasma membrane), and located too far from the plasma membrane to respond to changes in membrane voltage.

Having established the limitations that arise when comparing the AP waveform across different cells, we went on to explore the modulation of AP shape within the same neuron. By performing simultaneous GEVI imaging and current-clamp recordings before and after the addition of voltage-gated channel antagonists (30 $\mu \mathrm{m}$ of the $\mathrm{Kv}$ blocker 4 -ap and/or $40 \mathrm{~nm}$ of the Nav blocker TTX) we were able to subtly alter the AP waveform (Fig. 2). Application of these antagonists either together or separately created a large palette of AP waveforms, which we then compared with the waveforms of the corresponding electrophysiological measures. We found that both Ace-mNeon and Archon2 could faithfully report changes in AP kinetics (Fig. 2C, $D, F, G$ ), with relatively small measurement errors (Fig. 2I,J). More important, we now observed a strong correlation between AP amplitude measured optically and electrophysiologically (Fig. $2 E, H)$. Together, our data provides strong evidence that Ace$\mathrm{mNeon}$ and Archon2 can be used to measure AP kinetics reliably 
A

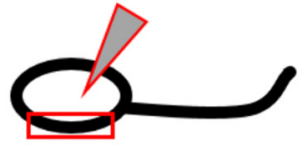

B

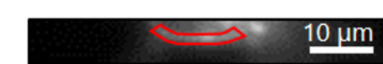

$\mathbf{B}^{\prime}$

$$
\text { Single trials }
$$

Ephys

GEVI

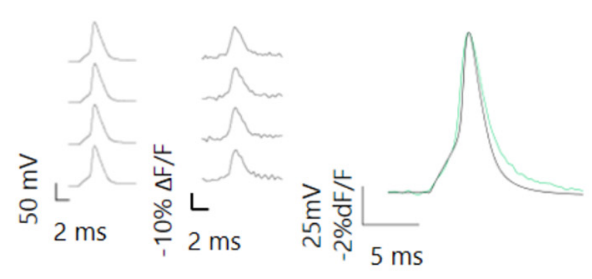

Average of 20

\section{E}

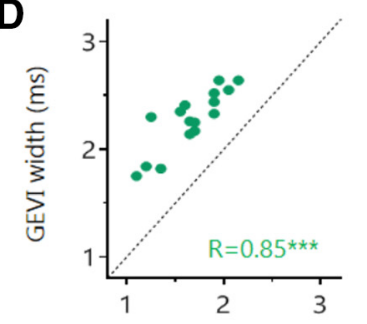

Ephys width (ms)

\section{G}

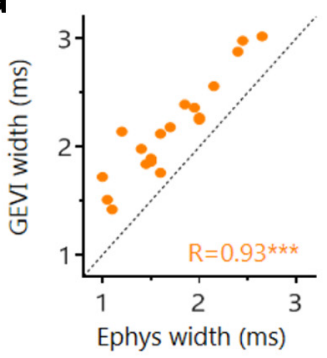

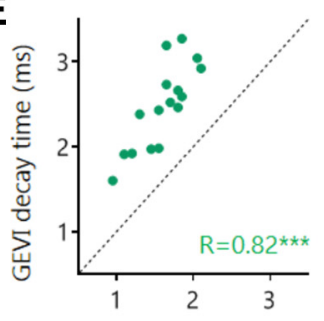

Ephys decay time (ms)

1

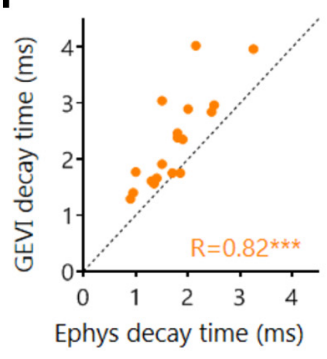

X20 repeats

C

Archon2

$\mathbf{C}^{\prime}$

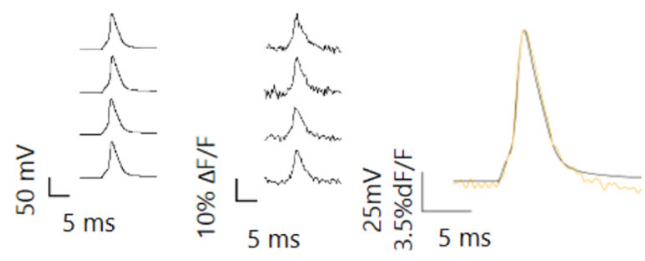

$\mathbf{F}$

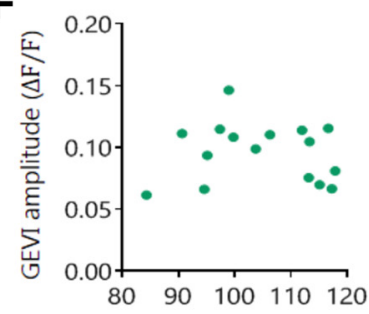

Ephys amplitude (mV)

I

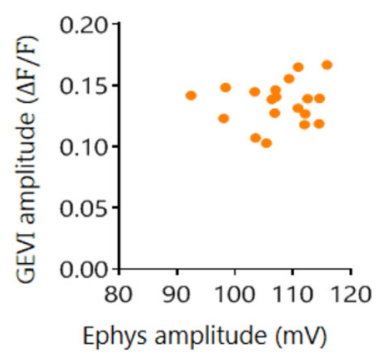

J

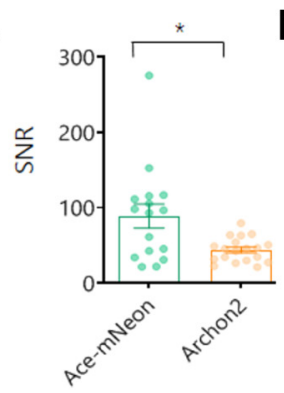

K

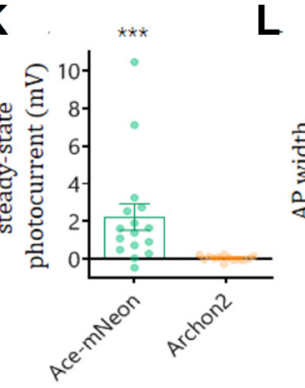

$\mathbf{L}$

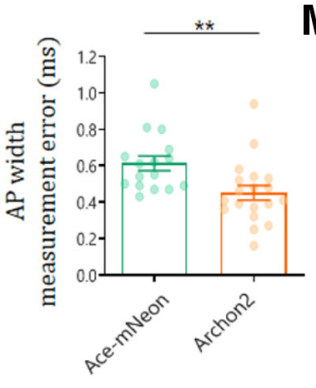

M

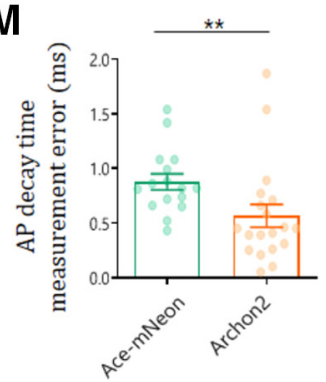

Figure 1. Optical and electrophysiological measure of the somatic AP waveform. A, Schematic representation of the experimental setup; neurons expressing a GEVI were held in whole-cell current clamp while simultaneously imaging a segment of the somatic membrane at room temperature. The stimulation protocol consisted in a time-locked current pulse delivered through the patch pipette $(\boldsymbol{I})$ to evoke a single AP, while subjecting the cells to LED or laser illumination (light) and high-speed camera acquisition at $3.2 \mathrm{kHz}$. The protocol was repeated 20 times, and the resulting recordings were averaged. $\boldsymbol{B}, \boldsymbol{C}$, Representative example cells expressing Ace-mNeon (B) and Archon2 (C), with selected ROls of the somatic membrane for optical trace analysis shown in red. $\boldsymbol{B}^{\prime}$, $\boldsymbol{C}^{\prime}$, Reference Ephys (left) and single-trial optical (center) recordings of evoked somatic APs acquired from the example cells in $\boldsymbol{B}$ and $\boldsymbol{C}$, respectively. Overlay of voltage and optical somatic AP recordings (right) for Ace-mNeon (green) and Archon 2 (orange). $\boldsymbol{D}-\boldsymbol{F}$, Evoked AP width $(\boldsymbol{D})$, decay time $(\boldsymbol{E})$, and amplitude $(\boldsymbol{F})$ were measured simultaneously with Ace-mNeon and reference Ephys recordings in the same cells to assess accuracy of GEVI measurements. $\mathbf{G}-\boldsymbol{I}$, Evoked AP width $(\boldsymbol{G})$, decay time $(\boldsymbol{H})$, and amplitude $(\boldsymbol{I})$ were measured simultaneously with Archon2 and reference Ephys recordings in the same cells to assess accuracy of GEVI measurements. J, SNR of optically recorded APs with the two GEVIs; ${ }^{*} p<0.05$; Kruskal-Wallis tests. $\boldsymbol{K}$, Steady-state photocurrent induced in response to GEVI illumination with the corresponding light source ( $505 \mathrm{~nm} \mathrm{LED} \mathrm{at} 10 \mathrm{~mW} / \mathrm{mm}^{2}$ for Ace-mNeon; $635 \mathrm{~nm}$ laser at $2.6 \mathrm{~W} / \mathrm{mm}^{2}$ for Archon2); ${ }^{* * *} p<0.001 ;$ one sample Wilcoxon test. $\boldsymbol{L}, \mathbf{M}$, Absolute AP width measurement error $(\boldsymbol{L})$ and decay time measurement error $(\boldsymbol{M})$ recorded with Ace-mNeon and Archon2 relative to reference Ephys recordings. Ace-mNeon, $N=16$ cells; Archon2, $N=19$ cells. $\boldsymbol{R}$, Pearson's correlation coefficient; $\boldsymbol{L}, \boldsymbol{M}$, Mann-Whitney tests; ${ }^{* * *} p<0.001,{ }^{* *} p<0.01$. All measurements performed on averages of 20 repeats. 
A

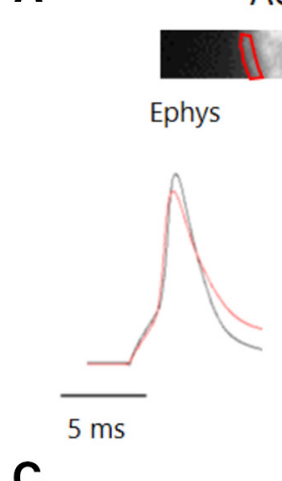

C

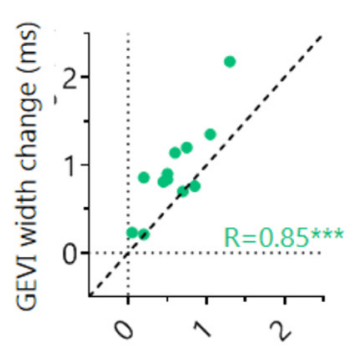

Ephys width change (ms)

F

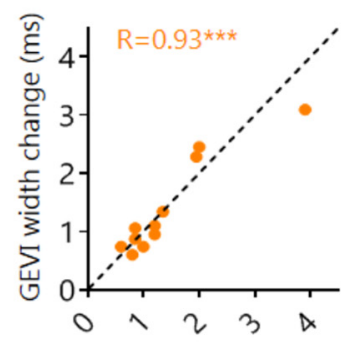

Ephys width change (ms)
B

$10 \mu \mathrm{m}$

Voltage imaging

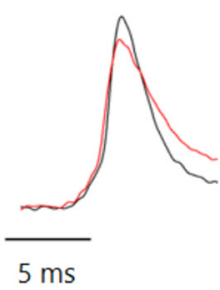

D

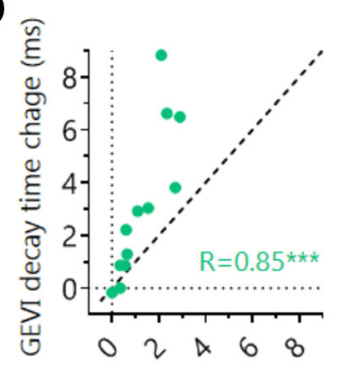

Ephys decay time change (ms)

G

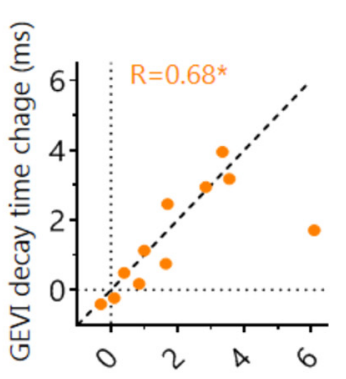

Ephys decay time change (ms)

E

$\mathrm{H}$
Archon2

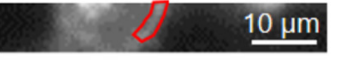

Ephys

Voltage imaging
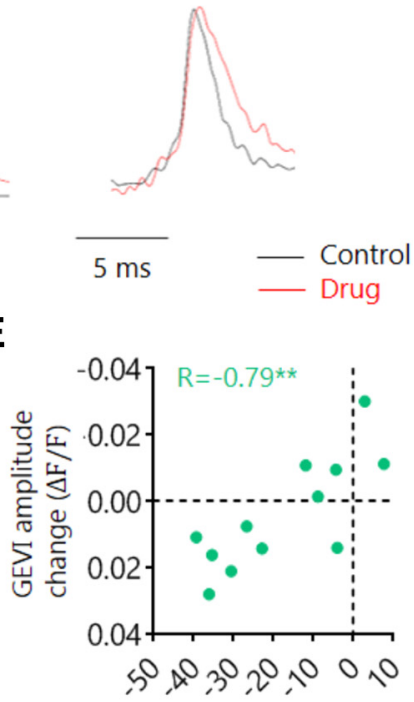

Ephys amplitude change (mV)

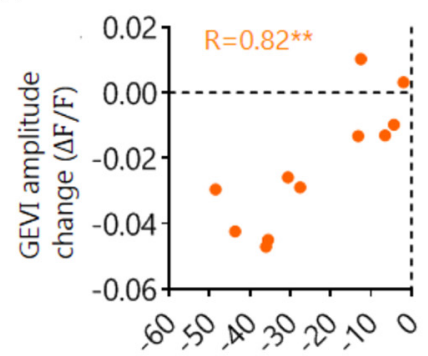

Ephys amplitude change (mV)
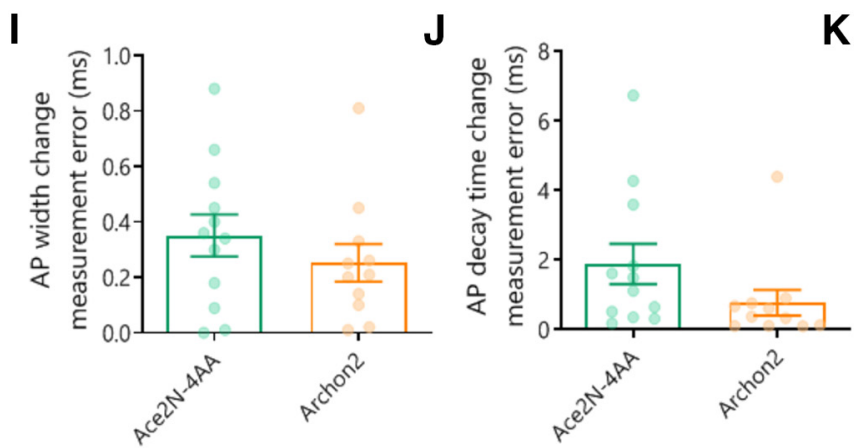

K

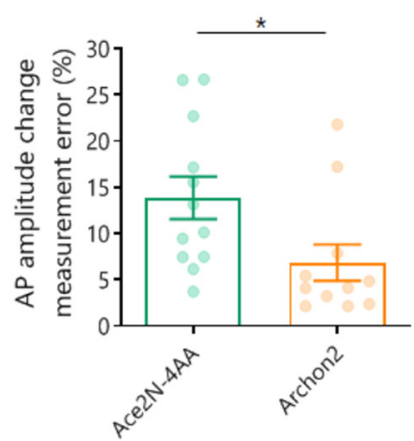

Figure 2. Optical and electrophysiological measure of drug-induced modulation of somatic AP waveform. $A, B$, Top, Imaging window with selected ROl of the somatic membrane shown in red. Bottom, average traces before and after addition of $30 \mu \mathrm{m}$ 4-ap and $40 \mathrm{~nm}$ TTX (drug), recorded with Ephys and Ace-mNeon/Archon2. (-E, Change in AP width (C), decay time (D), and amplitude $(\boldsymbol{E})$ induced by perfusion with drug, measured simultaneously with Ace-mNeon and Ephys in the same cells for comparison. $\boldsymbol{F}-\boldsymbol{H}$, Change in AP width $(\boldsymbol{F})$, decay time $(\boldsymbol{G})$, and amplitude $(\boldsymbol{H})$ induced by perfusion with drug, measured simultaneously with Archon2 and Ephys in the same cells for comparison. $\boldsymbol{I} \boldsymbol{K} \boldsymbol{K}$, Absolute measurement error of drug-induced modulation of AP width $(\boldsymbol{I})$, decay $(\boldsymbol{J})$, and amplitude $(\boldsymbol{K})$. Amplitude change was converted to percentage change to enable GEVI and Ephys comparison. Ace-mNeon, $N=12$ cells; Archon2, $N=11$ cells.

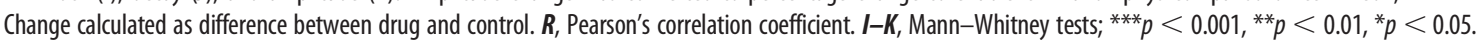

both within and across cells. AP amplitude, on the other hand, can only be reliably compared within the same membrane segment (Fig. 2K).

Reassured that both GEVIs were able to report changes in AP waveform at the soma with comparable accuracies, we went on to test whether it was possible to image the AP in the axons of dissociated CA1 pyramidal neurons. Compared with the soma, the plasma membrane area in the axon is much reduced, meaning far less GEVI molecules are available to be trafficked there and imaged. The result is relatively lower baseline fluorescence levels from background, which at high acquisition rates meant the GEVI signal was more vulnerable to camera noise (Popovic et al., 2015). At near physiological temperatures $\left(32^{\circ} \mathrm{C}\right)$ APs were elicited by somatic stimulation with a bipolar electrode, and both 
A

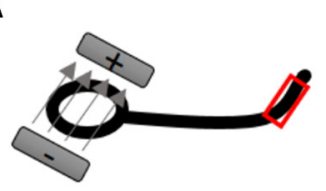

B
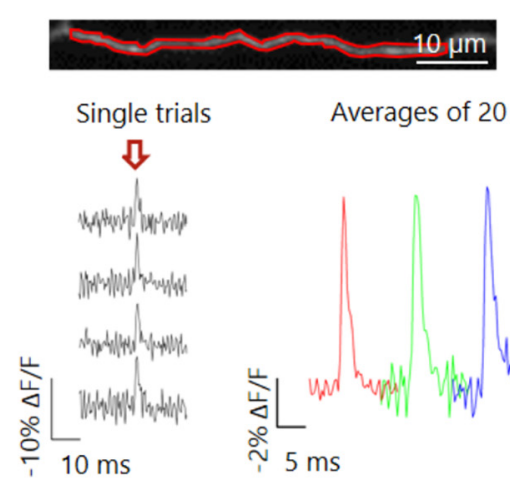

D

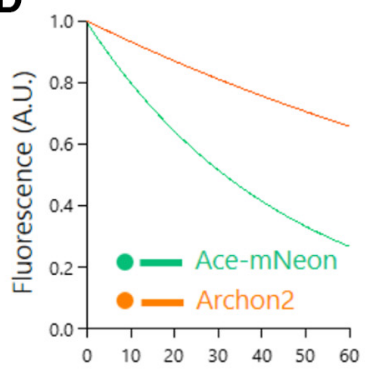

light exposure (s)

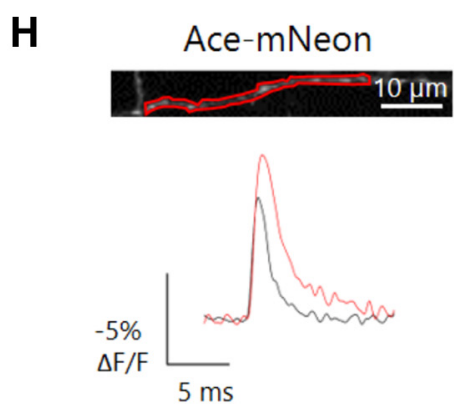

K

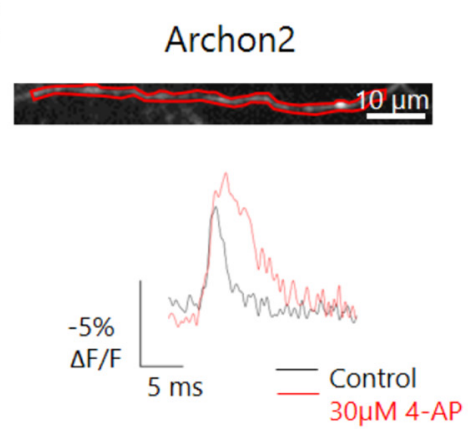

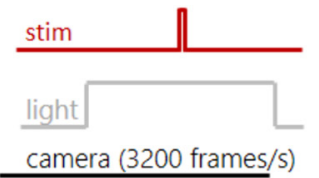

C Archon2
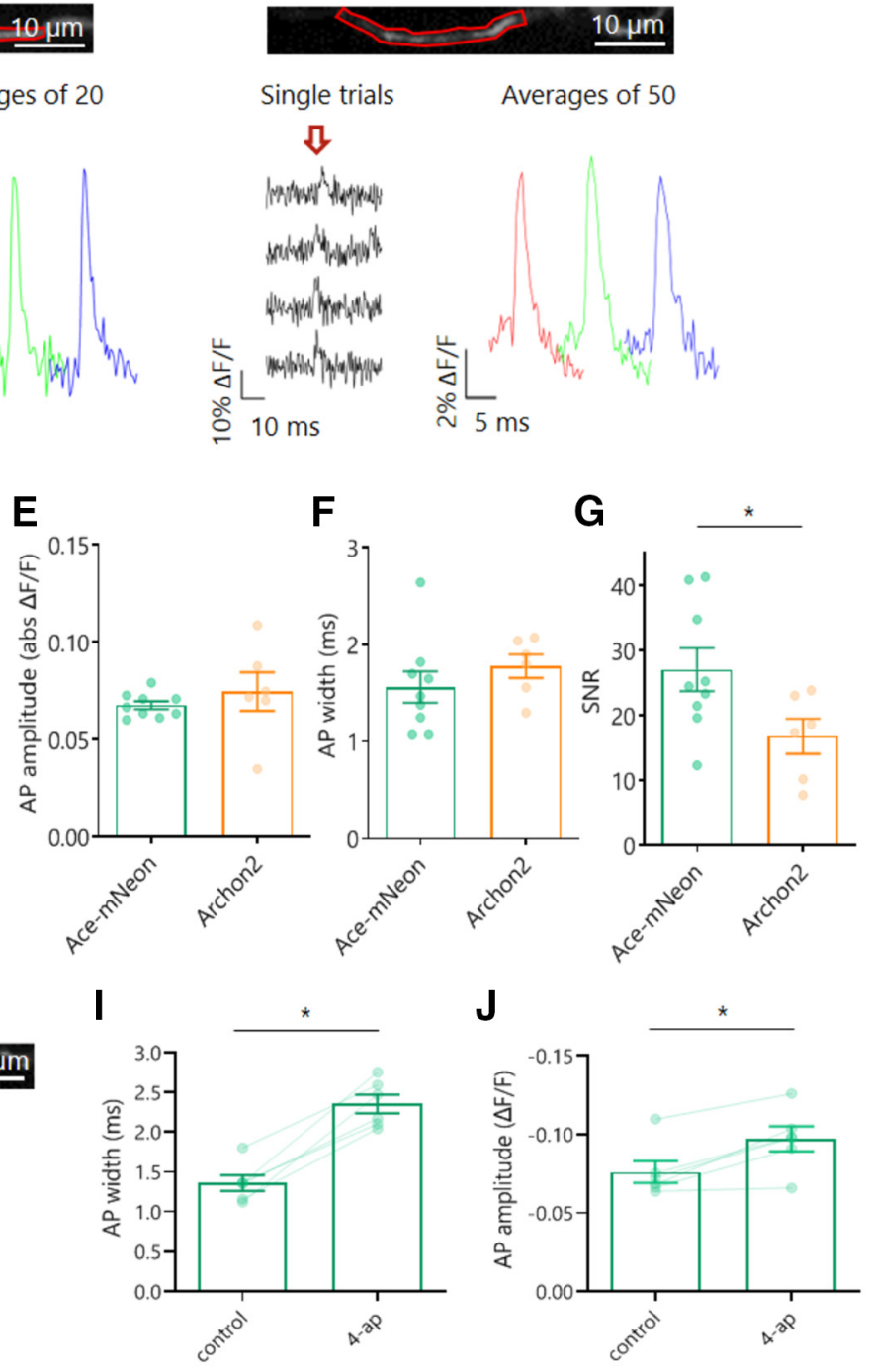

L
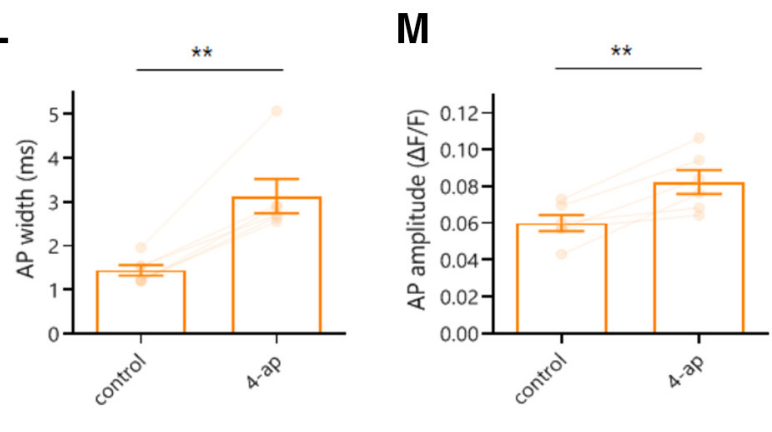

Figure 3. Voltage imaging of AP waveform in axons. A, Schematic representation of the experimental setup; neurons expressing Ace-mNeon or Archon2 were stimulated locally with a bipolar electrode while imaging a fragment of the axonal membrane. Time-locked 1 ms stimulation pulses of 10V were delivered to elicit single APs while subjecting the cells to LED or laser illumination and high-speed camera acquisition at $3.2 \mathrm{kHz}$. Experiments performed at near physiological temperature $\left(32^{\circ} \mathrm{C}\right) . \boldsymbol{B}, \boldsymbol{C}$, Top, Example axons from cells expressing Ace-mNeon $(\boldsymbol{A})$ and Archon2 $(\boldsymbol{B})$ with selected axonal ROls shown in red. Bottom, GEVI recordings obtained from the respective ROls, showing single trials as well as the averages over sequential groups of $20 / 50$ repeats within the same recording. The arrow indicates the timing of stimulation pulses. $\boldsymbol{D}$, Average bleaching curve of recordings with Ace-mNeon and Archon2 under exposure with $10 \mathrm{~mW} /$ $\mathrm{mm}^{2} 505$ LED light and $2.6 \mathrm{~W} / \mathrm{mm}^{2}$ laser light, respectively, normalized to initial fluorescence intensity. $\boldsymbol{E}$, GEVI signal magnitude in response to an evoked AP in the axon, expressed as the 
Ace-mNeon and Archon2-mediated responses were detected in single trials (Fig. 3A). To enhance the SNR we averaged across multiple repeats (from 20 to 50), with the number of repeats tailored to the levels of photobleaching for each probe (Fig. $3 B, C)$. When analyzing the baseline GEVI fluorescence over time, Ace-mNeon exhibited higher bleaching rates than Archon2, with time constants of $45.71 \mathrm{~s}$ and $143.62 \mathrm{~s}$, respectively (Fig. 3D). The axonal AP waveform was similar for both GEVIs (peak $\Delta \mathrm{F} / \mathrm{F}$ amplitude of $6.8 \pm 0.2 \%$ and $7.5 \pm 1 \%$, and an AP width of $1.56 \pm 0.16 \mathrm{~ms}$ and $1.78 \pm 0.12 \mathrm{~ms}$ for AcemNeon and Archon2, respectively; Fig. 3E,F; Mann-Whitney tests). In line with our somatic recordings (Fig. 1), the axonal AP width was larger than that previously reported by electrophysiological recordings at large boutons or axons, which typically ranged from 0.3 to $1.1 \mathrm{~ms}$, depending on neuron type (Geiger and Jonas, 2000; Kole et al., 2007; Vivekananda et al., 2017). Such differences likely result from the on/off kinetics of the respective GEVI. As expected from Figure 1, the SNR of AP signals from Ace-mNeon was larger than that of Archon2 (27.08 \pm 3.29 vs $16.82 \pm 2.7$; Fig. $3 G$; $p<0.05$; Mann-Whitney test).

We went on to test whether the temporal resolution of GEVI recordings in the axon was sufficient to detect AP waveform modulation. Neurons expressing either Ace-mNeon or Archon2 were stimulated locally with a bipolar electrode and imaged before and after perfusion with $30 \mu \mathrm{m}$ 4-ap to block Kv1 and Kv3 channels (Coetzee et al., 1999). We observed a clear widening of the axonal AP width on application of 4-ap, in agreement with previous findings (Kole et al., 2007; Shu et al., 2007; Alle et al., 2011). Notably, 4-ap also induced an increase in AP amplitude (Fig. 3H-M; $p<0.05$; Wilcoxon tests). Although this observation contrasts with the electrophysiological findings in the axons of cortical neurons (Kole et al., 2007), increases in AP amplitude have been observed in previous voltage imaging studies in hippocampal axons following blockade of the 4ap-sensitive Kv subfamilies (Hoppa et al., 2014; Cho et al., 2020).

To attain sufficient SNR to capture the axonal AP waveform with GEVIs, optical recordings were subjected to both temporal and spatial averaging. On the one hand, sets of 20 and 50 single trials were averaged to significantly increase SNR in recordings with Ace-mNeon and Archon2, respectively (Fig. 4A,E). We observed that despite the impact of loss of fluorescence over time on SNR, the recorded AP width and amplitude for both GEVIs remained constant for extended periods, allowing for several sets of repeats under different conditions to be performed in a single experiment (Fig. $4 B-D, F-H$ ). In addition to temporal averaging, spatial averaging by increasing ROI size also contributed to maximize SNR in axonal voltage recordings (Fig. $4 I, J)$ ). We saw that averaging over axonal ROIs of varying lengths was not sufficient to affect the AP shape because of the rapid propagation of the axonal AP (Fig. $4 K, L)$.

Overall, we found that Ace-mNeon and Archon2 could reliably report modulation of the AP waveform both in the soma

$\leftarrow$

absolute value of $\Delta F / F$ for the two GEVIs. $F$, Width of axonal APs recorded with the two GEVIs. G, SNR of axonal APs recorded with the two GEVIs. $\boldsymbol{H}, \boldsymbol{K}$, Top, Imaging window with selected ROI of the axonal membrane shown in red. Bottom, average Ace-mNeon $(\boldsymbol{H})$ and Archon2 $(\boldsymbol{K})$ traces before and after addition of $30 \mu \mathrm{m}$ 4-ap. $\boldsymbol{I}, \boldsymbol{J}$, Axonal AP width $(\boldsymbol{I})$ and amplitude $(\boldsymbol{J})$ measured with Ace-mNeon before and after addition of $30 \mu \mathrm{m}$ 4-ap. $\boldsymbol{L}, \boldsymbol{M}$, Axonal AP width $(\boldsymbol{L})$ and amplitude $(\boldsymbol{M})$ measured with Archon2 before and after addition of $30 \mu$ м 4-ap. $\boldsymbol{D}-\mathbf{G}$, Ace-mNeon, $N=9$ cells; Archon2, $N=6$ cells; Mann-Whitney tests; I, J, $\boldsymbol{L}, \boldsymbol{M}$, Ace-mNeon, $N=6$ cells; Archon2, $N=6$ cells; Wilcoxon tests; ${ }^{*} p<0.05$, ${ }^{* *} p<$ 0.01 . and in the axon. Although Archon2 showed some improved kinetic accuracy over Ace-mNeon in the somatic AP waveform measurements, the superior brightness and signal-to-noise of Ace-mNeon proved better suited for axonal high-speed voltage imaging.

\section{Differential regulation of AP shape and $\mathrm{Kv}$ activity in the distal axon}

Next, we performed voltage imaging experiments with Ace$\mathrm{mNeon}$ to explore the differences in AP waveform properties in the distal axon compared with the soma and proximal axonal regions within the same cells. Neurons were subjected to local stimulation using a tungsten bipolar electrode at near physiological temperature. The evoked AP waveform was imaged at three subcellular locations: the somatic membrane, a proximal region of the axon $(<100 \mu \mathrm{m}$ away from the soma) and a distal region of the axon $(>450 \mu \mathrm{m}$ away from the soma; Fig. $5 A-B)$. The passive propagation length constant has been found to be $\sim 450 \mu \mathrm{m}$ in mammalian nonmyelinated glutamatergic axons (Alle and Geiger, 2006; Shu et al., 2006), and therefore the AP waveform measurements we made from distal axons should have experienced little to no influence from the somatic compartment. Proximal axons, on the other hand, can be subject to somatic fluctuations in membrane potential because of the close coupling between the two compartments. Importantly, proximal axons will encompass the axon initial segment (AIS), a subcellular structure typically located between 20 and $60 \mu \mathrm{m}$ from the soma in excitatory hippocampal cells where the AP initiates (Meeks and Mennerick, 2007; Schmidt-Hieber et al., 2008).

The temporal resolution of Ace-mNeon recordings $(3.2 \mathrm{kHz})$ was sufficient to visualize the direction and speed of AP propagation based on latency differences between the peaks recorded at the three subcellular locations (Fig. $5 A-C$ ). The speed of orthodromic axonal AP propagation was calculated from a linear fit to the latency of the distal axonal AP relative to the soma as a function of the distance from the soma and estimated to be $383.2 \pm$ $53 \mu \mathrm{m} / \mathrm{ms}$ (Fig. 5 C). This result is consistent with previous estimates of active propagation speed in hippocampal cells at physiological temperature (Meeks and Mennerick, 2007). Notably, the AP latency for proximal axonal segments was negative, indicating that AP initiation occurred in the proximal axon, presumably at the AIS (Fig. 5C).

To test whether the AP waveform was uniform across different subcellular locations, the parameters of waveforms recorded in the soma, proximally and distally within the axon, were compared. Only kinetic measurements of the waveform were considered as amplitudes cannot be reliably compared across different membrane segments without calibration (Fig. $1 F, I$ ). When comparing the AP waveform across different compartments, we found that the AP was sharper in distal axons compared with the soma or proximal axons. The mean AP width decreased with distance along the axon, ranging from $1.75 \pm 0.05 \mathrm{~ms}$ in the proxi$\mathrm{mal}$ axon and $1.72 \pm 0.06 \mathrm{~ms}$ in the soma to $1.53 \pm 0.05 \mathrm{~ms}$ in the distal axon (Fig. 5D). This difference was significant between proximal and distal axon waveforms and reached near statistical significance between distal axon and soma (Tukey's multiple comparisons test after one-way repeated measures ANOVA; $p<$ 0.01 and $p=0.056$, respectively). Similar differences were observed in the $80-20 \%$ decay of the AP (Fig. $5 E ; 2.73 \pm 0.2 \mathrm{~ms}$, $2.28 \pm 0.2 \mathrm{~ms}$ and $1.63 \pm 0.1 \mathrm{~ms}$ for soma, proximal, and distal axon, respectively; Tukey's multiple comparisons test after oneway repeated measures ANOVA; $p<0.001$ for somatic versus proximal and somatic vs distal comparisons; $p<0.05$ for 
A
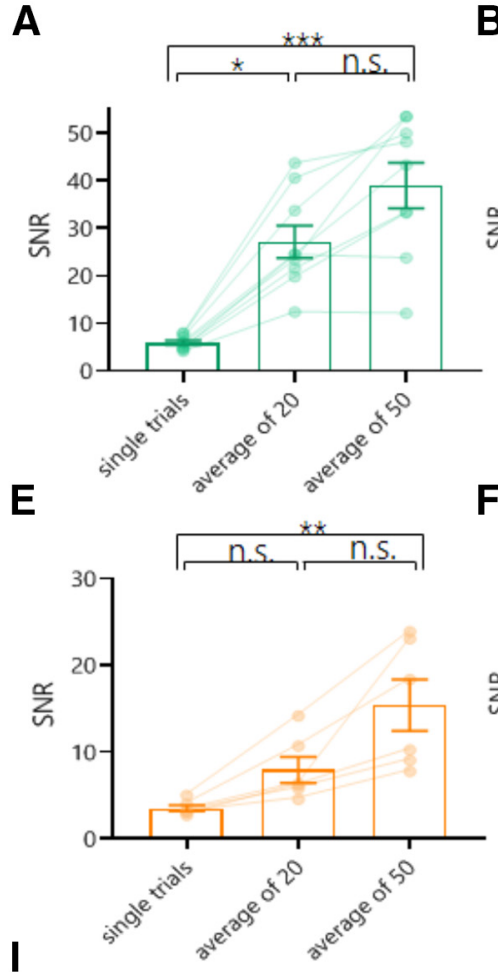

I

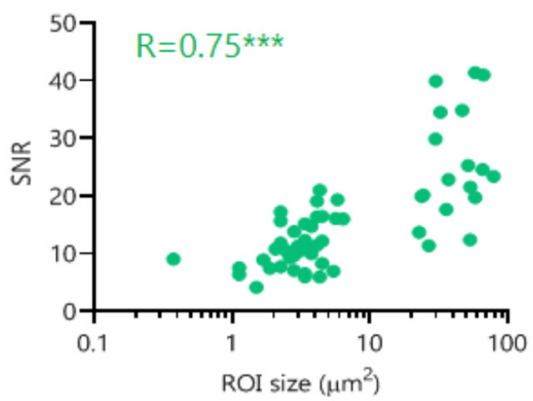

B

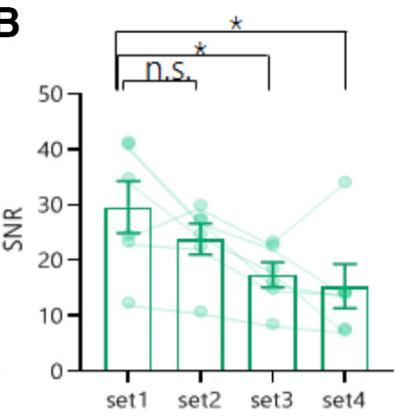

C

F

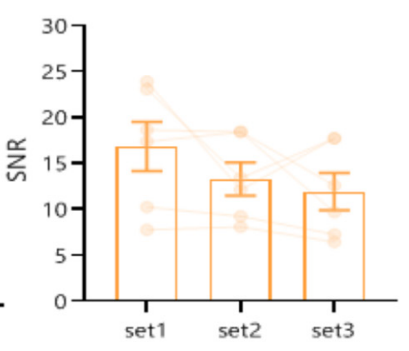

D

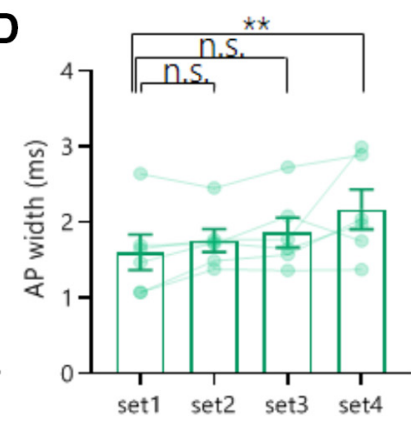

G

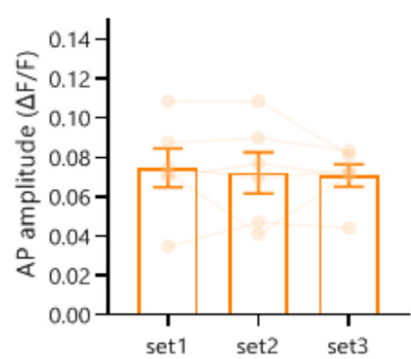

H

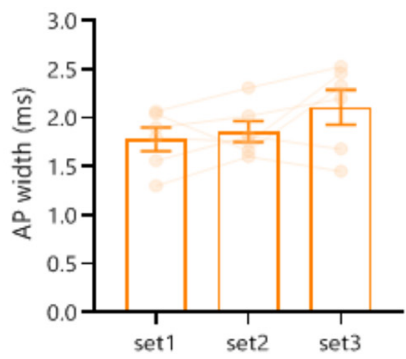

J

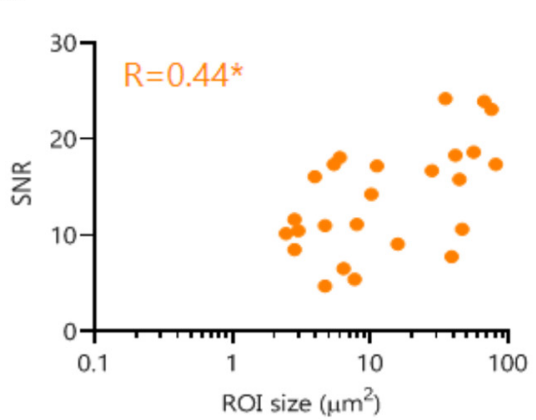

K

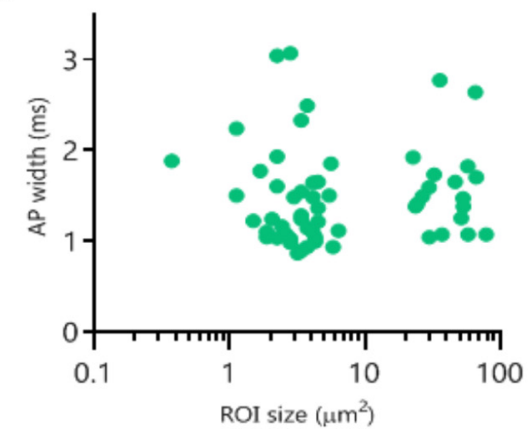

L
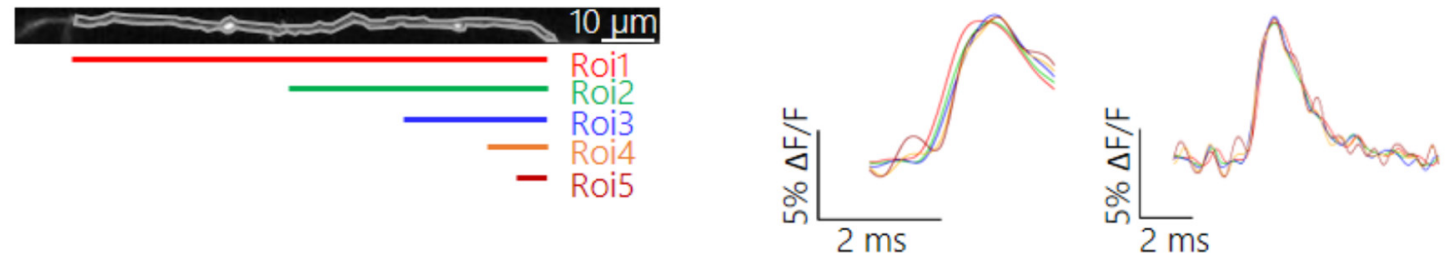

Figure 4. Impact of temporal and spatial averaging on SNR and consistency of axonal AP waveform GEVI recordings. $A$, SNR values taken from average recordings of increasing numbers of repeats performed with Ace-mNeon. $\boldsymbol{B}-\boldsymbol{D}$, AP SNR $(\boldsymbol{B})$, amplitude $(\boldsymbol{C})$, and width $(\boldsymbol{D})$ values measured on average recordings of sequential sets of 20 repeats performed with Ace-mNeon. $\boldsymbol{E}$, SNR values taken from average recordings of increasing numbers of repeats performed with Archon2. $\boldsymbol{F}-\boldsymbol{H}$, AP SNR $(\boldsymbol{F})$, amplitude $(\boldsymbol{G})$, and width $(\boldsymbol{H})$ values measured on average recordings of sequential sets of 50 repeats performed with Archon2. $\boldsymbol{I}, \boldsymbol{J}$, Ace-mNeon $(\boldsymbol{I})$ and Archon2 $(\boldsymbol{J})$ AP recording SNR values plotted against the size of the analyzed axonal ROI. $\boldsymbol{K}$, AP width recorded with Ace-mNeon plotted against the analyzed ROI size. $L$, Right, Example of an imaged axonal fragment and five varying size ROI selections along its length. Middle, AP rise average profiles for ROls 1-5, illustrating delays in the rise following the direction of AP propagation. Left, AP average profiles for ROIs 1-5 aligned to peak do not show alteration of the AP waveform because of averaging over large sections of the axon. For $\boldsymbol{A}, \mathbf{E}, N=9$ cells (Ace-mNeon), $N=6$ cells (Archon2); Kruskal-Wallis test with post hoc Dunn's multiple comparisons. $\boldsymbol{B}-\boldsymbol{D}, \boldsymbol{F}-\boldsymbol{H}, \boldsymbol{N}=6$ cells, Friedman tests with post hoc Dunn's multiple comparisons. $\boldsymbol{I}-\boldsymbol{K}$, Ace-mNeon, $N=56$ varying size Rols from 9 cells; Archon2, $N=25$ varying size ROls from 6 cells. $\boldsymbol{R}$, Spearman's correlation coefficient. ${ }^{*} p<0.05,{ }^{* *} p<0.01,{ }^{* * *} p<0.001$; n.S., Not significant.

proximal vs distal comparison). In contrast, within the distal axon, comparison of daughter and mother branches at bifurcation points did not show significant differences in width or 80$20 \%$ decay time across the different axonal branches (data not shown).

The differences in AP kinetics observed between the somatic and axonal compartments suggested that there might be underlying differences in the channels that shape the AP in the different subcellular compartments. We tested the contribution of the potassium channels susceptible to block by low concentrations of 4-ap, previously shown to be targeted specifically to the axon (Kole et al., 2007; Shu et al., 2007). APs were elicited by bipolar electrode stimulation in neurons expressing Ace-mNeon, and recordings were acquired at the soma, proximal, and distal axon before and after bath perfusion with $30 \mu \mathrm{M} 4$-ap (Fig. 5F). To control for changes in the recorded AP waveform that might 


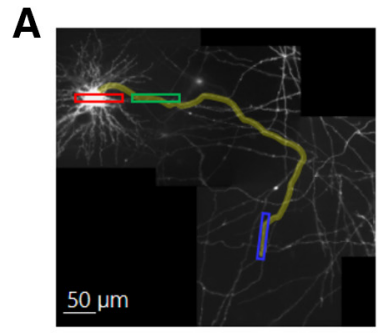

B

C

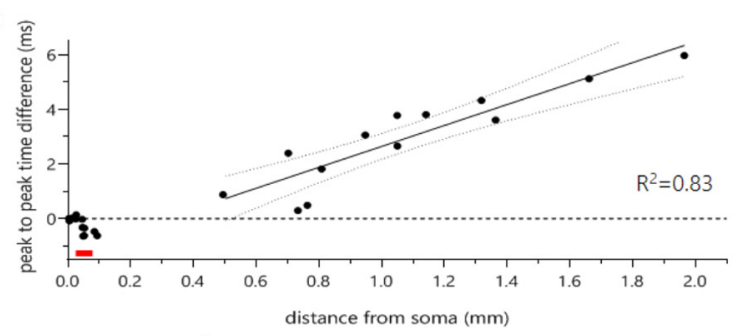

D

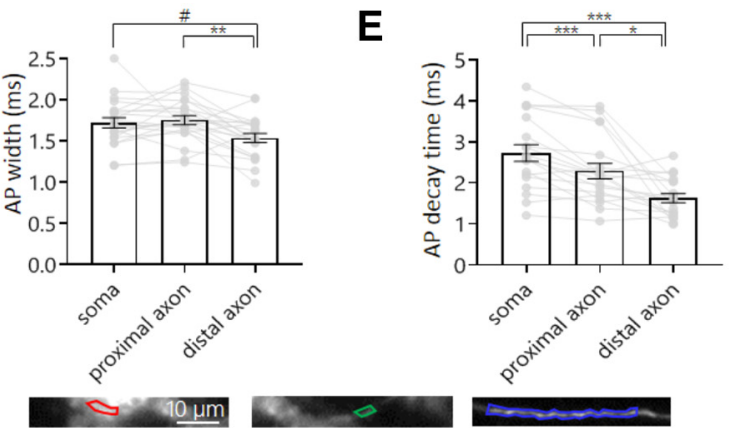

$\mathbf{F}$
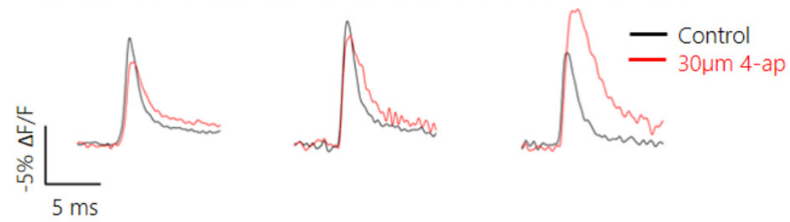

G

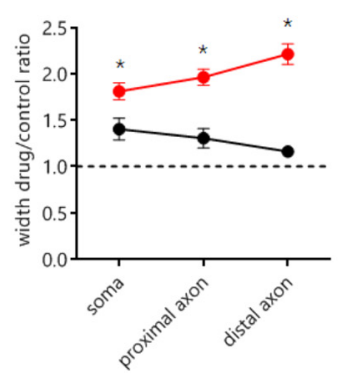

H

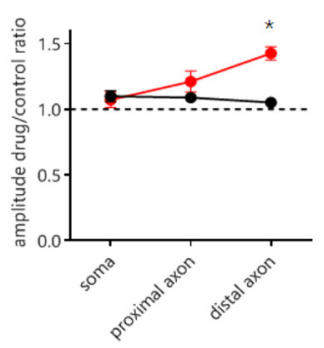

Figure 5. Differential regulation of AP shape by Kv channels along different subcellular compartments. A, Reconstructed mosaic of fluorescent images of the axonal arbor of an example neuron expressing Ace-mNeon, acquired at $60 \times$ magnification. Neurons were stimulated locally with a bipolar electrode, and ROls were chosen to include a portion of the somatic membrane, a proximal and a distal segment of the axon. Highlighted in yellow, the axonal path followed up to the most distal imaged fragment of the axon. Red, green, and blue rectangles indicate locations selected for imaging, enlarged in $\boldsymbol{B}$. $\boldsymbol{B}$, Imaged sections of the somatic membrane, a region of the axon proximal to the soma, and a region of the axon distal to the soma, respectively. The ROls drawn around membrane fragments that were selected to extract the fluorescent profile in time are shown for each image. Bottom, overlaid averages of optical recordings of time-locked evoked APs extracted from ROls containing the somatic membrane (red), the proximal axon (green) and the distal axon (blue). The red arrow indicates the time point of stimulation with the bipolar electrode. $\mathbf{C}$, Time difference between the AP peaks recorded in the soma and in the axon plotted against the distance of the axon ROls from the soma. Fit, linear regression performed with data from distal axon segments only. Red line indicates approximate location of the AIS. $\boldsymbol{D}, \boldsymbol{E}, \mathrm{AP}$ width (D) and $80-20 \%$ decay time $(\boldsymbol{E})$ recorded at the three subcellular locations within the same set of cells. $\boldsymbol{F}$, Average AP profile recorded with Ace-mNeon in the soma (top), proximal (middle), and distal (bottom) axon of the same cell. Traces shown for both the control condition occur within the experiment because of bleaching or phototoxicity, neurons from the same culture were imaged before and after perfusion with HBS without drug (mock). The results showed a strong 4-ap-induced broadening in all subcellular compartments, with the biggest effect on the distal axon (Fig. 5G; $p<0.05$ for mock versus 4 -ap comparison in all subcellular compartments; Mann-Whitney tests with Holm-Bonferroni correction). Interestingly, we also observed an increase in AP amplitude with respect to the mock-only control but only in distal axons (Fig. 5H; $p<0.05$; amplitude increase of $32 \%$ with respect to mock treatment; Mann-Whitney tests with Holm-Bonferroni correction). Together, our data show that 4-ap-sensitive $\mathrm{Kv}$ channels play a bigger role in controlling AP shape in distal rather than proximal axons and suggests that either the activity or spatial distribution of $\mathrm{Kv}$ channels is biased toward distal axonal domains.

\section{The distal axon was resilient to frequency-dependent AP broadening}

AP broadening during high-frequency trains is a form of AP waveform plasticity that has been described both in the soma (Connors et al., 1982; Shao et al., 1999; Faber and Sah, 2003) and in large presynaptic boutons of glutamatergic neurons (Jackson et al., 1991; Geiger and Jonas, 2000). More recently, whole-cell recordings from small cortical presynaptic boutons showed AP broadening was exclusive to glutamatergic, but not GABAergic, axons (Ritzau-Jost et al., 2021). Here, we went on to test whether the observed differences in AP properties and Kv channel activity across the axonal and somatic subcompartments result in differential modulation of the AP waveform under stimulation trains at different frequencies. Excitatory hippocampal neurons expressing Ace-mNeon were stimulated at $20 \mathrm{~Hz}$ with a bipolar electrode (Fig. 6A), and optical recordings were obtained from the soma, proximal, and distal axon segments (Fig. 6B-D). Although we observed a broadening of the AP during the train in both the soma and proximal axon (22 and 20\% increase in width of fifth AP relative to first AP, respectively), this effect was much smaller in distal axons (only a $4 \%$ increase in width; Fig. $6 E)$. Although some significant differences in AP amplitude were observed, they were generally small ( $<4 \%$ changes) and unlikely to have an important functional impact. As the modulation of AP waveform is typically frequency dependent (Jackson et al., 1991; Geiger and Jonas, 2000), we also measured distal AP shape in response to high-frequency bursts delivered at $200 \mathrm{~Hz}$. At such high frequencies, AP failures become much more likely (Raastad and Shepherd, 2003), so we only took recordings when we were certain that APs were fired to all stimuli. We found a broadening of the AP in distal axons at these high frequencies that was comparable to that observed in proximal axons at lower frequencies. Our results show that although distal axons were more resilient to changes in AP waveform, they were capable of

(black) and after perfusion with $30 \mu \mathrm{m}$ 4-ap (red). G, Quantification of the effect of 4ap (red) and mock (black) perfusion on the AP width for soma, proximal, and distal axon. The width increases observed were $38 \%(\mathrm{Cl}=6 \%, 70 \%), 62 \%(\mathrm{Cl}=26 \%$, $102 \%)$ and $95 \%(\mathrm{Cl}=85 \%, 148 \%)$ with respect to mock treatment, respectively. $\boldsymbol{H}$, Quantification of the effect of 4-ap (red) and mock (black) perfusion on the AP amplitude for soma, proximal, and distal axon. The amplitude increase observed in the distal axon was $32 \%$ (Cl 27\%, 57\%) with respect to mock treatment. D, E, N = 19 cells, one-way repeated measures ANOVA with post hoc Tukey's multiple comparisons test. $\boldsymbol{G}, \boldsymbol{H}, 4$-ap, $N=8$ cells; control, $N=4$ cells; Mann-Whitney tests with HolmBonferroni correction; \#p $<0.06,{ }^{*} p<0.05,{ }^{* *} p<0.01,{ }^{* * *} p<0.001$. 
A
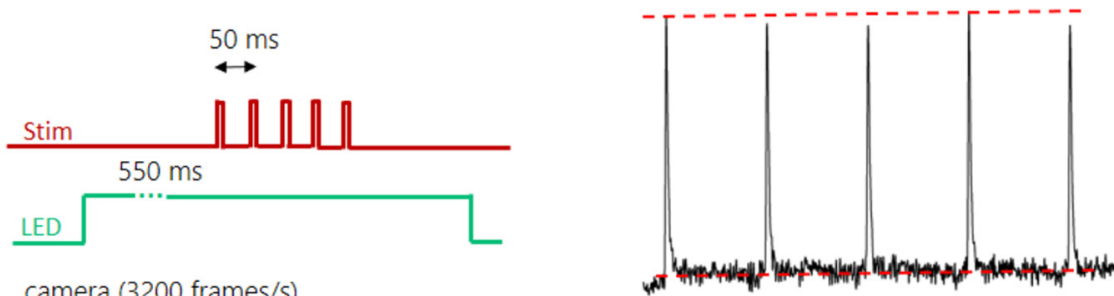

B

C

D

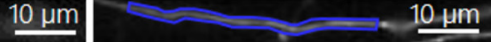

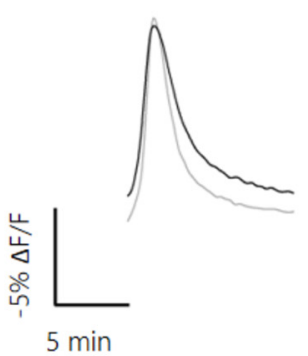

$\mathbf{E}$

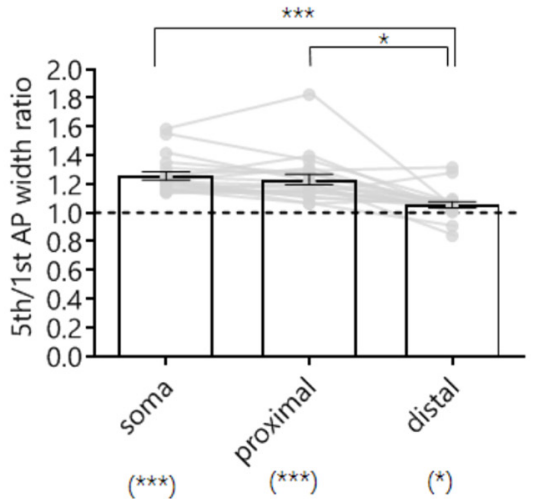

G

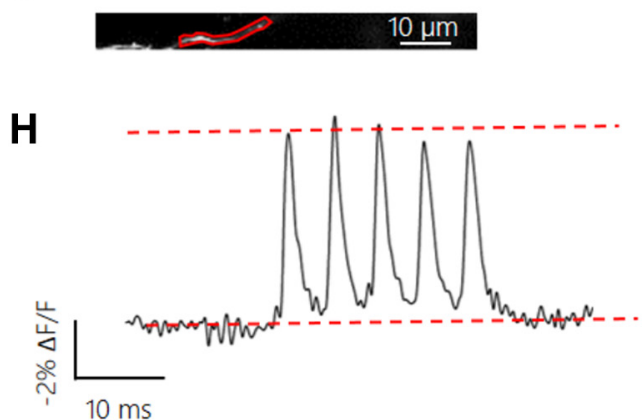

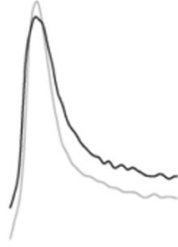
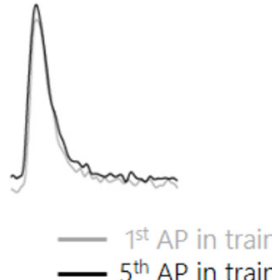

$\mathbf{F}$

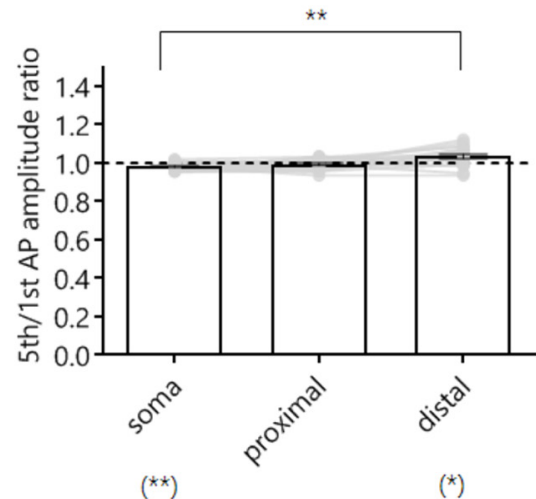

I

$\mathbf{J}$
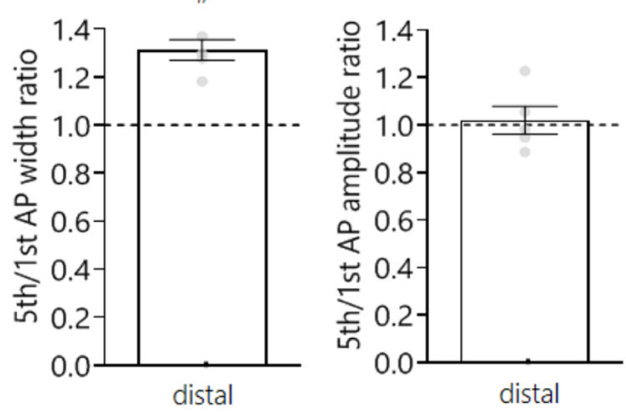

Figure 6. Frequency-dependent plasticity of AP waveform along different subcellular compartments. $A$, Schematic representation of the experimental setup; five pulse stimulation trains were delivered with an interstimulus interval (ISI) of $50 \mathrm{~ms}$ while subjecting the cell to continuous LED illumination and camera acquisition. The first and fifth APs were compared. $\boldsymbol{B}-\boldsymbol{D}$, Top, example neuron expressing Ace-mNeon with selected ROls in the soma $(\boldsymbol{B})$, proximal $(\boldsymbol{C})$, and distal axon segments $(\boldsymbol{D})$. Bottom, the respective average AP optic profiles of the first and fifth APs of the train overlaid. $\boldsymbol{E}$, Quantification of the width ratio between the fifth and first AP of the $20 \mathrm{~Hz}$ train in the different subcellular compartments. The widening observed in the soma, proximal, and distal axon was $22 \%(\mathrm{Cl}=17.01$, $27.27 \%), 20 \%(C=14.02,26.95 \%)$ and $4 \%(C l=2.34,6.98 \%)$ increase in width of the fifth AP relative to the first, respectively. $F$, Quantification of the amplitude ratio between the fifth and first AP of the $20 \mathrm{~Hz}$ train in the different subcellular compartments. The somatic AP exhibited a decrease of $1.7 \%$ in its amplitude $(\mathrm{Cl}=-3.01,-0.72 \%)$, whereas the AP peak in the distal axon increased by $3.8 \%(\mathrm{Cl}=1.0,6.95 \%)$. G, Example axon expressing Ace-mNeon with selected $\mathrm{ROI}$ in red, from a neuron that was subjected to $200 \mathrm{~Hz}$ train simulation. $\boldsymbol{H}$, Average optic trace of a $200 \mathrm{~Hz} 5$-AP train recorded from the cell in $A$. I, Quantification of the fifth to first AP width ratio. An increase of $29.7 \%$ of the fifth relative to the first AP was observed $(\mathrm{Cl}=18.18,43.58 \%)$. J, Quantification of the fifth to first AP amplitude ratio. $E, F, N=20$ cells; above the graphs, Friedman test with Dunn's post hoc multiple comparisons; below the graphs, one sample Wilcoxon tests with Holm-Bonferroni correction. $I, J, N=5$ cells; one sample Wilcoxon tests; ${ }^{*} p<0.05,{ }^{* *} p<0.01,{ }^{* * *} p<0.001, \# p=0.0625$.

short-term forms of plasticity when pushed to higher frequencies. This difference in frequency tuning may also have interesting functional consequences for synaptic transmission along different axonal compartments.
Pharmacological blockade of Kv1 and Kv3 channels led to an increase in frequency-dependent AP plasticity

Our results suggest the existence of a distance-dependent difference in the short-term plasticity of AP waveform. Previous 
reports have implicated $\mathrm{Kv}$ channel inactivation in the broadening of APs during high-frequency trains in axonal boutons (Jackson et al., 1991; Geiger and Jonas, 2000). Because we showed that $\mathrm{Kv}$ channels played a role in controlling AP width in distal axons, we next investigated whether they also played a role in controlling AP shape during a train. Imaging of Ace-mNeon was conducted in the soma and axonal domains in response to a train of APs delivered at $20 \mathrm{~Hz}$, before and after perfusion of either $30 \mu \mathrm{m} 4$-ap or a mock control (Fig. 7A-D). As expected, the first AP in a burst increased in width following application of 4-ap, and this widening was more pronounced in distal axons. Surprisingly, however, subsequent APs broadened even further during the train, a feature that was observed along all compartments, including distal axons (Fig. 7E; $p<0.05$ for all comparisons; Wilcoxon matchedpairs signed rank tests with HolmBonferroni correction for this and the subsequent panels). There was no change in frequency-dependent broadening in the mock perfused cells (Fig. 7G). Finally, we observed no change in amplitude modulation on perfusion with 4-ap or mock (Fig. $7 F, H)$.

These results suggest that 4-apsensitive Kv channels play a role in ensuring AP waveform stability. The increased contribution of $\mathrm{Kv}$ channels to shaping the AP waveform in the distal axon might therefore constitute a mechanism to guarantee faithful axonal AP propagation and reduce AP waveform plasticity.

\section{Discussion}

In this study we have demonstrated that Ace-mNeon and Archon2 were able to report the AP waveform with high fidelity, and in so doing, have uncovered a role for $\mathrm{Kv}$ channels in controlling the shape of APs in distal axonal domains.

By performing ground truth experiments to compare somatic AP GEVI recordings with whole-cell patch-clamp recordings, we showed that it was possible to capture the AP accurately and reliably using both Ace-mNeon and Archon2. Quantification of the AP waveform under control conditions and on drug-induced modulation of the AP shape showed that within the same cell, changes in both AP kinetics and amplitude could be detected with GEVIs and were highly correlated to the changes observed with

E

G correction.
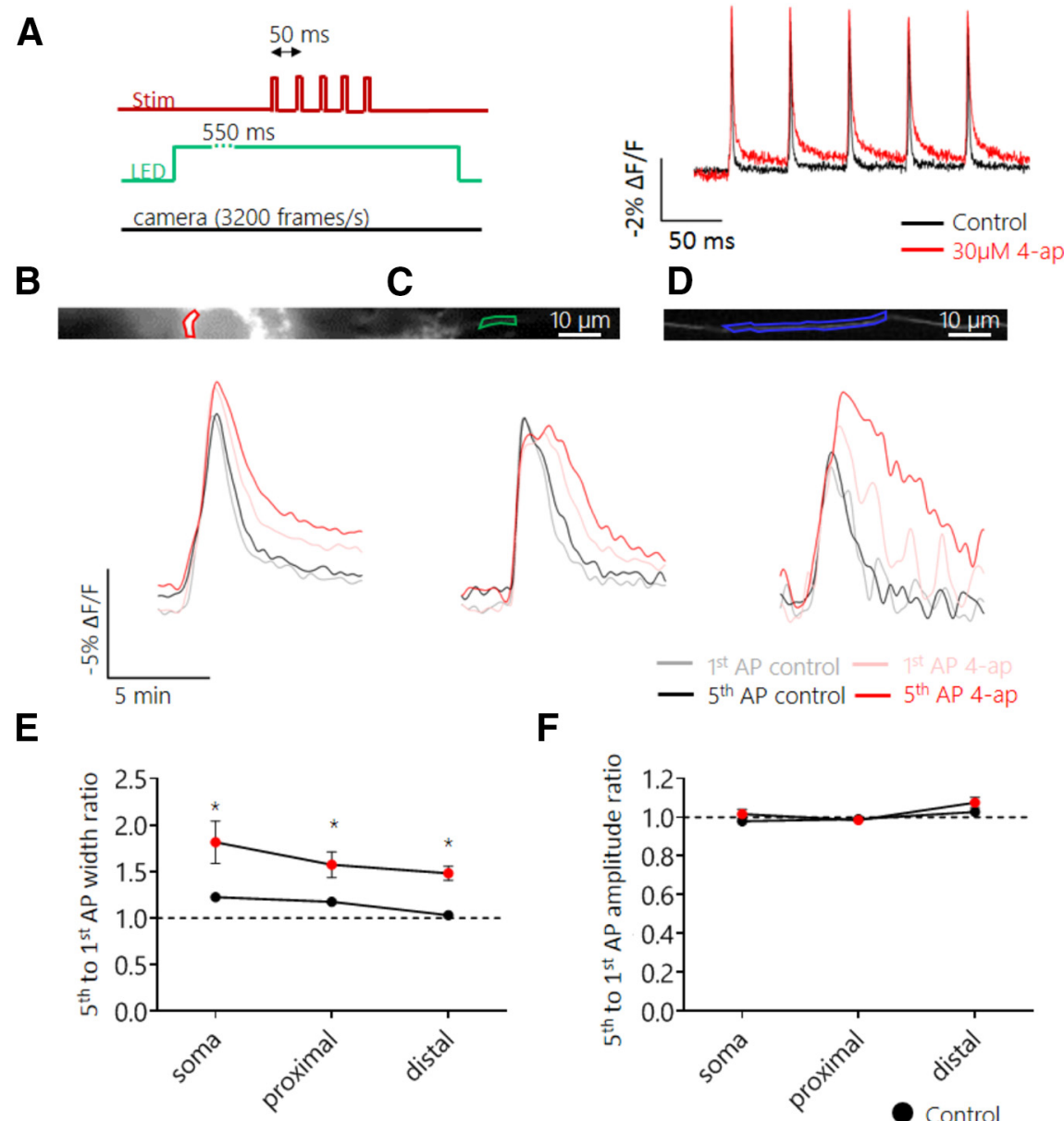

$\mathbf{F}$

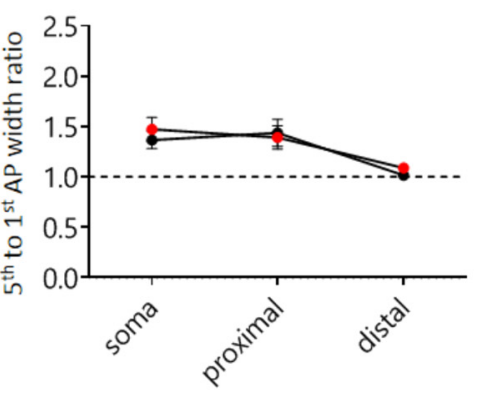

$\mathbf{H}$
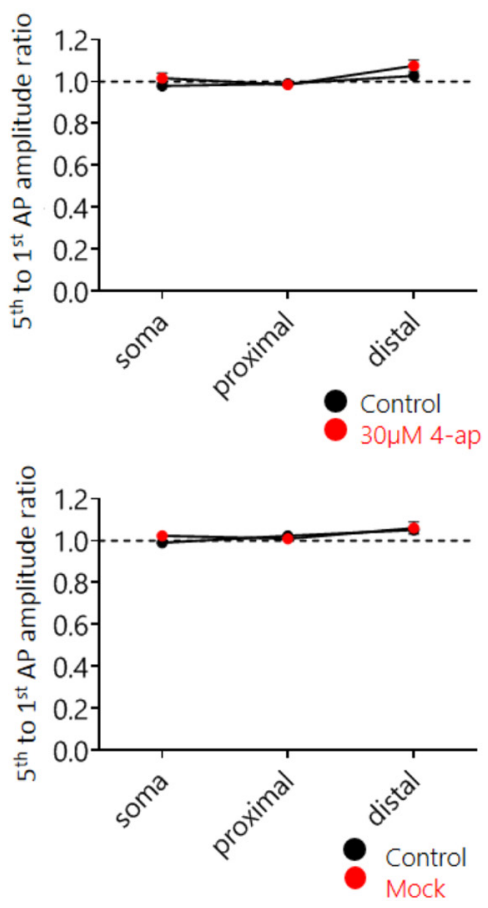

Figure 7. Block of 4-ap-sensitive Kv channels increased AP broadening during $20 \mathrm{~Hz}$ trains across all subcellular compartments. $A$, Schematic representation of the experimental setup; five pulse stimulation trains were delivered with an ISI of $50 \mathrm{~ms}$ while subjecting the cell to continuous LED illumination and camera acquisition. Cells were recorded before and after addition of $30 \mu \mathrm{m}$ 4-ap. $\boldsymbol{B}-\boldsymbol{D}$, Top, Example neuron expressing Ace-mNeon with selected ROls in the soma $(\boldsymbol{B})$, proximal $(\boldsymbol{C})$, and distal axon segments (D). Bottom, overlaid average AP optic profiles of the first and fifth APs of the train before and after addition of $30 \mu \mathrm{m} \mathrm{4-}$ ap for the respective ROls. $\boldsymbol{E}, \boldsymbol{F}$, Quantification of the width $(\boldsymbol{E})$ and amplitude $(\boldsymbol{F})$ ratio between the fifth and first AP of the $20 \mathrm{~Hz}$ train in the three subcellular compartments, before and after addition of $30 \mu \mathrm{m}$ 4-ap. AP width facilitation increased by $30.50 \%$ relative to the control recordings in the soma $(\mathrm{Cl}=10.70,154.9 \%)$, by $28.87 \%$ in the proximal axon $(\mathrm{Cl}=2.0,105.0 \%)$, and by $39.51 \%$ in the distal axon $(\mathrm{Cl}=11.47,88.15 \%) . \boldsymbol{G}, \boldsymbol{H}$, Quantification of the width $(\boldsymbol{G})$ and amplitude $(\boldsymbol{H})$ ratio between the fifth and first AP of the $20 \mathrm{~Hz}$ train in the three subcellular compartments, before and after perfusion with mock (HBS without 4-ap). $\boldsymbol{E}, \boldsymbol{F}, \boldsymbol{N}=8$ cells; $\boldsymbol{G}, \boldsymbol{H}, \boldsymbol{N}=4$ cells. ${ }^{*} p<0.05$; Wilcoxon matched-pairs signed rank tests with Holm-Bonferroni

electrophysiology. However, when comparing across different cells, only differences in AP kinetics, not amplitude, remained correlated. The most likely explanation for this is the local variation in GEVI expression, and membrane targeting, across different cells or subcellular compartments. Measures of AP 
amplitude for comparison across different cells cannot be obtained by normalizing responses to resting GEVI fluorescence as this value may not accurately represent the levels of GEVI on the membrane. Indeed, other normalizing approaches are needed to obtain absolute membrane voltage measures (Hoppa et al., 2014). As a result, only relative changes in voltage amplitude, within the same subcellular compartment, can be compared accurately. When comparing the AP kinetics measured with Archon 2 or Ace-mNeon, we found that although both sensors overestimated the AP duration, Archon2 was temporally more accurate. The most likely explanation for this difference is the faster reported on/off kinetics of Archon2 compared with Ace-mNeon (Gong et al., 2015; Piatkevich et al., 2018).

Compared with the soma, the ability of GEVIs to reliably report the AP waveform in the axon was much more limited by the SNR. At such low brightness levels, camera dark noise starts contributing significantly to measurement error (Popovic et al., 2015). In effect, GEVI AP width measurements were largely overestimated when compared with patch-clamp data from similar boutons (Geiger and Jonas, 2000; Vivekananda et al., 2017), even when imaged with the fast Archon2 sensor. However, both indicators were able to measure AP width and amplitude modulation on addition of 4 -ap, even in bouton-size ROIs $\left(\sim 1 \mu \mathrm{m}^{2}\right)$. Remarkably, the temporal resolution attained by voltage imaging with Ace-mNeon performed at a frame rate of $\sim 3 \mathrm{kHz}$ was sufficient to place AP initiation in the proximal axon and measure AP conduction velocity.

Overall, we found that the accuracy with which an AP waveform can be captured with GEVIs is affected by the filtering imposed by sensor kinetics and acquisition speed. However, the limiting factor for subcellular voltage imaging is SNR, which is in turn determined by sensor brightness and sensitivity (Popovic et al., 2015). Although we found both sensors equivalent in terms of resolution and stability, there was a practical advantage in using Ace-mNeon because of its higher brightness and SNR, conducive to a higher success rate of the recordings even in cultures with variable transfection efficiency.

Taking advantage of the unique spatial resolution of GEVI imaging, we monitored the AP in three subcellular locations within hippocampal excitatory neurons: the somatic membrane, a proximal axon region ( $<100 \mu \mathrm{m}$ from the soma) that generally encompassed the AIS, and a distal axon region $(>450 \mu \mathrm{m}$ from the soma). We observed heterogeneity in both the AP waveform and its plasticity during a train in different subcellular compartments, which paralleled the modulation of $\mathrm{Kv}$ channels in these compartments. The AP repolarization phase was found to be shortened in the distal axon relative to the soma and proximal axon, a feature that has also been observed in other neurons including layer 5 cortical pyramidal neurons and granule cells of the dentate gyrus (Geiger and Jonas, 2000; Kole et al., 2007). Furthermore, we found a lower susceptibility to frequency-dependent modulation in the distal axon than in the soma or the proximal axon region. Although this behavior has been described in cortical and CA3 neurons (Meeks et al., 2005; Kole et al., 2007), there is evidence to the contrary in CA1 neurons, where spike broadening during a train has been shown to increase with distance from the soma (Kim, 2014). In our experiments, only high-frequency stimulation $(200 \mathrm{~Hz})$ resulted in the broadening of the distal axon AP, a feature that may endow the distal axon with high-pass filtering properties. It has been proposed that axonal AP broadening provides a potential mechanism to modulate neurotransmission and increase the encoding capacity of the axon (Geiger and Jonas, 2000; Shu et al., 2006). However, the limited AP broadening in the distal axon compared with the soma observed here suggests that reliable conduction is prioritized in the axon. Maintaining a sharp AP during trains could ensure a timely membrane voltage repolarization, minimizing the inactivation of Nav channels and protecting the axon from failures (Gründemann and Clark, 2015).

The pharmacological experiments shown here revealed differential effects of 4-ap-sensitive $\mathrm{Kv}$ channels in shaping the AP along different subcellular compartments. Kv1 and Kv3 channel subtypes, susceptible to block by the low concentration of 4-ap used here, have previously been shown to control AP waveform in the axon (Kole et al., 2007; Shu et al., 2007; Boudkkazi et al., 2011; Foust et al., 2011; Hoppa et al., 2014; Kim, 2014; Rowan et al., 2014; Cho et al., 2020; Ritzau-Jost et al., 2021). We found that blocking Kv channels caused AP broadening across all compartments (soma and axon) but that the effect was strongest in distal axons, suggesting Kv channels may be preferentially targeted (or be preferentially activated) at these distal domains. Although all studies agree that blocking $\mathrm{Kv}$ channels broadens the axonal AP, the effect on AP amplitude is more controversial. A recent study using voltage imaging has shown increases in AP amplitude in the axon and implicated $\mathrm{Kv}$ channels in blunting the AP depolarization (Hoppa et al., 2014). However, other studies, using mainly electrophysiology, have not observed any changes in AP amplitude following similar manipulations (Geiger and Jonas, 2000; Kole et al., 2007; Alle et al., 2011; Ritzau-Jost et al., 2021). Although the reason for this discrepancy is not clear, and may be methodological, it is possible that differences in neuron type and position along the axon influence the outcome. Indeed, our results show changes in AP amplitude occur only in distal axons, suggesting that knowledge of axon position is a crucial parameter when assessing the role of Kvs on AP waveform.

One surprising finding from our experiments was that the block of 4-ap-sensitive Kv channels increased AP broadening during a train in all compartments measured (soma as well as proximal and distal axon). Usually, spike broadening is thought to occur through the gradual inactivation of Kv channels as the spike train progresses (Jackson et al., 1991; Ma and Koester, 1996; Geiger and Jonas, 2000; Kim et al., 2005), which may be mediated by the $\operatorname{Kv} 1 \beta$ subunit (Cho et al., 2020). Here, however, we see that following the initial increase in AP width following 4ap application, the subsequent APs broaden further during the train. These results suggest that under basal conditions the current mediated by 4 -ap-sensitive channels occludes the inactivating current that would otherwise contribute to use-dependent AP broadening. In other words, Kv channels act to stabilize AP waveform from other destabilizing currents. The fact that distal axons are more sensitive to 4 -AP suggest that $\mathrm{Kv}$ channels are likely responsible for maintaining AP shape fidelity and invariance in these subcellular compartments.

The stability of the AP is particularly important in the distal axon, where up to $70 \%$ of the Nav current is inactivated (Engel and Jonas, 2005; Schmidt-Hieber and Bischofberger, 2010), and the likelihood of AP failure increases with every crossed branch point (Lüscher and Shiner, 1990). Although a tighter control of AP kinetics might be an efficient mechanism for maintaining signal fidelity, it would be of great interest to investigate what impact it has on axonal signal processing and neurotransmitter release. A narrower AP might result in less voltage-gated calcium channel activation per AP in distal presynaptic boutons than in proximal ones. Furthermore, the differential properties of activity-dependent AP waveform plasticity in proximal and distal axon regions likely translate into further differences in neuronal 
output in proximal and distal boutons. Not only is the proximal axon prone to frequency-dependent AP broadening, as shown in this study, recent studies suggest that it is also susceptible to AP waveform modulation by subthreshold signals that propagate from the soma (Rama et al., 2018). Therefore, the encoding capabilities of proximal and distal regions of the axon are likely very different. Experiments with simultaneous imaging of voltage and neurotransmitter release would reveal whether these signal processing differences translate into region-specific differences in neurotransmitter release.

\section{References}

Alle H, Geiger JRP (2006) Combined analog and action potential coding in hippocampal mossy fibers. Science 311:1290-1293.

Alle H, Kubota H, Geiger JRP (2011) Sparse but highly efficient Kv3 outpace BKCa channels in action potential repolarization at hippocampal mossy fiber boutons. J Neurosci 31:8001-8012.

Boudkkazi S, Fronzaroli-Molinieres L, Debanne D (2011) Presynaptic action potential waveform determines cortical synaptic latency. J Physiol 589:1117-1131.

Branco T, Staras K (2009) The probability of neurotransmitter release: variability and feedback control at single synapses. Nat Rev Neurosci 10:373383.

Chéreau R, Saraceno GE, Angibaud J, Cattaert D, Nägerl UV (2017) Superresolution imaging reveals activity-dependent plasticity of axon morphology linked to changes in action potential conduction velocity. Proc Natl Acad Sci U S A 114:1401-1406.

Cho IH, Panzera LC, Chin M, Hoppa MB (2017) Sodium channel $\beta 2$ subunits prevent action potential propagation failures at axonal branch points. J Neurosci 37:9519-9533.

Cho IH, Panzera LC, Chin M, Alpizar SA, Olveda GE, Hill RA, Hoppa MB (2020) The potassium channel subunit $\operatorname{Kv} \beta 1$ serves as a major control point for synaptic facilitation. Proc Natl Acad Sci U S A 117:2993729947.

Coetzee WA, Amarillo YIMY, Chiu J, Chow A, Lau D, McCormack T, Morena H, Nadal MS, Ozaita A, Pountney D, Saganich M, Miera EV-S, Rudy B (1999) Molecular diversity of K+ channels. Ann N Y Acad Sci 868:233-255.

Connors BW, Gutnick MJ, Prince DA (1982) Electrophysiological properties of neocortical neurons in vitro. J Neurophysiol 48:1302-1320.

Debanne D, Campanac E, Bialowas A, Carlier E, Alcaraz G (2011) Axon physiology. Physiol Rev 91:555-602.

Dittman JS, Ryan TA (2019) The control of release probability at nerve terminals. Nat Rev Neurosci 20:177-186.

Engel D, Jonas P (2005) Presynaptic action potential amplification by voltage-gated $\mathrm{Na}+$ channels in hippocampal mossy fiber boutons. Neuron 45:405-417.

Faber ESL, Sah P (2003) Ca2+-activated K+ (BK) channel inactivation contributes to spike broadening during repetitive firing in the rat lateral amygdala. J Physiol 552:483-497.

Foust AJ, Yu Y, Popovic M, Zecevic D, McCormick DA (2011) Somatic membrane potential and Kv1 channels control spike repolarization in cortical axon collaterals and presynaptic boutons. J Neurosci 31:1549015498.

Geiger JRP, Jonas P (2000) Dynamic control of presynaptic Ca2+ inflow by fast-inactivating $\mathrm{K}+$ channels in hippocampal mossy fiber boutons. Neuron 28:927-939.

Goldstein SS, Rall W (1974) Changes of action potential shape and velocity for changing core conductor geometry. Biophys J 14:731-757.

Gong Y, Huang C, Li JZ, Grewe BF, Zhang Y, Eismann S, Schnitzer MJ (2015) High-speed recording of neural spikes in awake mice and flies with a fluorescent voltage sensor. Science 350:1361-1366.

Gründemann J, Clark BA (2015) Calcium-activated potassium channels at nodes of ranvier secure axonal spike propagation. Cell Rep 12:17151722.

Hochbaum DR, Zhao Y, Farhi SL, Klapoetke N, Werley CA, Kapoor V, Zou P, Kralj JM, Maclaurin D, Smedemark-Margulies N, Saulnier JL, Boulting GL, Straub C, Cho YK, Melkonian M, Wong GK-S, Harrison DJ, Murthy VN, Sabatini BL, Boyden ES, et al. (2014) All-optical electrophysiology in mammalian neurons using engineered microbial rhodopsins. Nat Methods 11:825-833.

Hoppa MB, Gouzer G, Armbruster M, Ryan TA (2014) Control and plasticity of the presynaptic action potential waveform at small CNS nerve terminals. Neuron 84:778-789.

Jackson MB, Konnerth A, Augustine GJ (1991) Action potential broadening and frequency-dependent facilitation of calcium signals in pituitary nerve terminals. Proc Natl Acad Sci U S A 88:380-384.

Jiang M, Chen G (2006) High Ca 2+ -phosphate transfection efficiency in low-density neuronal cultures. Nat Protoc 1:695-700.

Kawaguchi S-Y (2019) Dynamic factors for transmitter release at small presynaptic boutons revealed by direct patch-clamp recordings. Front Cell Neurosci 13:269.

Kawaguchi S-Y, Sakaba T (2015) Control of inhibitory synaptic outputs by low excitability of axon terminals revealed by direct recording. Neuron 85:1273-1288.

Kawaguchi S-Y, Sakaba T (2017) Fast Ca2+ buffer-dependent reliable but plastic transmission at small CNS synapses revealed by direct bouton recording. Cell Rep 21:3338-3345.

Khaliq ZM, Raman IM (2005) Axonal propagation of simple and complex spikes in cerebellar Purkinje neurons. J Neurosci 25:454-463.

Kim J, Wei DS, Hoffman DA (2005) Kv4 potassium channel subunits control action potential repolarization and frequency-dependent broadening in rat hippocampal CA1 pyramidal neurones. J Physiol 569:41-57.

Kim S (2014) Action potential modulation in CA1 pyramidal neuron axons facilitates OLM interneuron activation in recurrent inhibitory microcircuits of rat hippocampus. PLoS One 9:e113124.

Knöpfel T, Song C (2019) Optical voltage imaging in neurons: moving from technology development to practical tool. Nat Rev Neurosci 20:719-727.

Kole MHP, Letzkus JJ, Stuart GJ (2007) Axon initial segment Kv1 channels control axonal action potential waveform and synaptic efficacy. Neuron 55:633-647.

Kralj JM, Douglass AD, Hochbaum DR, MacLaurin D, Cohen AE (2011) Optical recording of action potentials in mammalian neurons using a microbial rhodopsin. Nat Methods 9:90-95.

Lüscher HR, Shiner JS (1990) Computation of action potential propagation and presynaptic bouton activation in terminal arborizations of different geometries. Biophys J 58:1377-1388.

Ma M, Koester J (1996) The role of K+ currents in frequency-dependent spike broadening in Aplysia R20 neurons: a dynamic-clamp analysis. J Neurosci 16:4089-4101

Meeks JP, Mennerick S (2007) Action potential initiation and propagation in CA3 pyramidal axons. J Neurophysiol 97:3460-3472.

Meeks JP, Jiang X, Mennerick S (2005) Action potential fidelity during nor$\mathrm{mal}$ and epileptiform activity in paired soma-axon recordings from rat hippocampus. J Physiol 566:425-441.

Monsivais P, Clark BA, Roth A, Häusser M (2005) Determinants of action potential propagation in cerebellar purkinje cell axons. J Neurosci 25:464-472.

Neher E, Sakaba T (2008) Multiple roles of calcium ions in the regulation of neurotransmitter release. Neuron 59:861-872.

Novak P, Gorelik J, Vivekananda U, Shevchuk AI, Ermolyuk YS, Bailey RJ, Bushby AJ, Moss GWJ, Rusakov DA, Klenerman D, Kullmann DM, Volynski KE, Korchev YE (2013) Nanoscale-targeted patch-clamp recordings of functional presynaptic ion channels. Neuron 79:1067-1077.

Panzera LC, Hoppa MB (2019) Genetically encoded voltage indicators are illuminating subcellular physiology of the axon. Front Cell Neurosci 13:1-9.

Piatkevich KD, Jung EE, Straub C, Linghu C, Park D, Suk H-J, Hochbaum DR, Goodwin D, Pnevmatikakis E, Pak N, Kawashima T, Yang C-T, Rhoades JL, Shemesh O, Asano S, Yoon Y-G, Freifeld L, Saulnier JL, Riegler C, Engert F, et al. (2018) A robotic multidimensional directed evolution approach applied to fluorescent voltage reporters article. Nat Chem Biol 14:352-360.

Popovic M, Vogt K, Holthoff K, Konnerth A, Salzberg BM, Grinvald A, Antic SD, Canepari M, Zecevic D (2015) Imaging submillisecond membrane potential changes from individual regions of single axons, dendrites and spines. Adv Exp Med Biol 859:57-101.

Raastad M, Shepherd GMG (2003) Single-axon action potentials in the rat hippocampal cortex. J Physiol 548:745-752.

Rama S, Zbili M, Bialowas A, Fronzaroli-Molinieres L, Ankri N, Carlier E, Marra V, Debanne D (2015) Presynaptic hyperpolarization induces a fast 
analogue modulation of spike-evoked transmission mediated by axonal sodium channels. Nat Commun 6:10163.

Rama S, Zbili M, Debanne D (2018) Signal propagation along the axon. Curr Opin Neurobiol 51:37-44.

Ritzau-Jost A, Tsintsadze T, Krueger M, Ader J, Bechmann I, Eilers J, Barbour B, Smith SM, Hallermann S (2021) Large, stable spikes exhibit differential broadening in excitatory and inhibitory neocortical boutons. Cell Rep 34:108612.

Rowan MJM, Tranquil E, Christie JM (2014) Distinct Kv channel subtypes contribute to differences in spike signaling properties in the axon initial segment and presynaptic boutons of cerebellar interneurons. J Neurosci 34:6611-6623.

Sabatini BL, Regehr WG (1997) Control of neurotransmitter release by presynaptic waveform at the granule cell to Purkinje cell synapse. J Neurosci 17:3425-3435.

Sasaki T, Matsuki N, Ikegaya Y, Sasakit T, Matsuki N, Ikegaya Y (2012) Targeted axon-attached recording with fluorescent patch-clamp pipettes in brain slices. Nat Protoc 7:1228-1234.

Scarnati MS, Clarke SG, Pang ZP, Paradiso KG (2020) Presynaptic calcium channel open probability and changes in calcium influx throughout the action potential determined using AP-waveforms. Front Synaptic Neurosci 12:17.
Schmidt-Hieber C, Jonas P, Bischofberger J (2008) Action potential initiation and propagation in hippocampal mossy fibre axons. J Physiol 586:18491857.

Schmidt-Hieber C, Bischofberger J (2010) Fast sodium channel gating supports localized and efficient axonal action potential initiation. J Neurosci 30:10233-10242.

Schneggenburger R, Neher E (2000) Intracellular calcium dependence of transmitter release rates at a fast central synapse. Nature 406:889-9893.

Shao LR, Halvorsrud R, Borg-Graham L, Storm JF (1999) The role of BKtype $\mathrm{Ca} 2+$-dependent $\mathrm{K}+$ channels in spike broadening during repetitive firing in rat hippocampal pyramidal cells. J Physiol 521:135-146.

Shu Y, Hasenstaub A, Duque A, Yu Y, McCormick DA (2006) Modulation of intracortical synaptic potentials by presynaptic somatic membrane potential. Nature 441:761-765.

Shu Y, Yu G, Yang J, McCormick DA (2007) Selective control of cortical axonal spikes by a slowly inactivating $\mathrm{K}+$ current. Proc Natl Acad Sci U S A 104:11453-11458.

Thévenaz P, Ruttimann UE, Unser M (1998) A pyramid approach to subpixel registration based on intensity. IEEE Trans Image Process 7:27-41.

Vivekananda U, Novak P, Bello OD, Korchev YE, Krishnakumar SS, Volynski KE, Kullmann DM (2017) Kv1.1 channelopathy abolishes presynaptic spike width modulation by subthreshold somatic depolarization. Proc Natl Acad Sci U S A 114:2395-2400. 